\title{
Omics strategies for revealing Yersinia pestis virulence
}

\section{Ruifu Yang*, Zongmin Du, Yanping Han, Lei Zhou, Yajun Song, Dongsheng Zhou and Yujun Cui}

Beijing Institute of Microbiology and Epidemiology, Beijing, China

\section{Edited by:}

Matthew Francis, Umeå University, Sweden

\section{Reviewed by:}

Vladimir L. Motin, University of

Texas Medical Branch, USA

Brett Chromy, University of

California Davis School of Medicine, USA

\section{*Correspondence:}

Ruifu Yang, Beijing Institute of

Microbiology and Epidemiology,

No. 20, Dongdajie, Fengtai,

Beijing 100071, China.

e-mail: ruifuyang@gmail.com
Omics has remarkably changed the way we investigate and understand life. Omics differs from traditional hypothesis-driven research because it is a discovery-driven approach. Mass datasets produced from omics-based studies require experts from different fields to reveal the salient features behind these data. In this review, we summarize omics-driven studies to reveal the virulence features of Yersinia pestis through genomics, trascriptomics, proteomics, interactomics, etc. These studies serve as foundations for further hypothesis-driven research and help us gain insight into $Y$. pestis pathogenesis.

Keywords: Yersinia pestis, omics, virulence, genomics, transomics
Omics is a collective concept of high-throughput studies for understanding life (Morrison et al., 2006) using the integrative strategies of genomics, proteomics, transcriptomics, and metabolomics, as well as the newly developed omics strategies of RNomics (Bhattacharjee et al., 2012), lipidomics (Hartler et al., 2012), kinomics (Kindrachuk et al., 2012), glycomics (Turnbull and Sasisekharan, 2010), peptomics (Olsen et al., 2002), antigenomics (Levesque et al., 2012), chemomics (Wang et al., 2008), etc. A detailed discussion on omics can be found in the Wikipedia website (http://en.wikipedia.org/wiki/Omics). The development of high-throughput technologies often yields large datasets that require extensive bioinformatic integration to apply omics in biological research. Combining omics strategies to elucidate specific features of an organism has become a trend, providing a unique opportunity to gain holistic understanding of the physiological and pathological characterization of the studied organism. The word "trans-omics" is also used to describe this type of studies (Tuohy et al., 2009; Yang et al., 2011b; Ogata et al., 2012). A multitude of extensive reviews introducing different omics technologies exist (Morrison et al., 2006; Kolker, 2009; Holmes et al., 2010; Knox, 2010; Mahapatra, 2010; Ning and Lo, 2010; Wild, 2010), and we do not intend to repeatedly introduce these concepts and their related techniques in this review. We aim to provide a summary of the applications of omics strategies in studying Yersinia pestis, particularly in revealing its virulence.

\section{GENOMICS FOR UNCOVERING $Y$. pestis VIRULENCE-ASSOCIATED GENES}

Some fragments or genes in $Y$. pestis, such as plasmids (pCD1, pMT1, and pPCP1) and chromosomal loci (pgm locus and pH6 antigen coding genes), have already been identified as virulence-associated even before its genome was decoded (Perry and Fetherston, 1997). The first genome of Y. pestis (strain CO92) offered unprecedented opportunities for understanding the virulence traits of this deadly pathogen (Parkhill et al., 2001).
In addition, two other $Y$. pestis strain whole genomes, KIM and 91001, were decoded (Lindler et al., 1998; Perry et al., 1998; Deng et al., 2002; Song et al., 2004). These findings provided the opportunity to uncover virulence-associated genes due to the avirulent nature of 91001 in humans through comparative genomics (Zhou et al., 2004a).

\section{VIRULENCE GENES COMMON IN Y. pestis AND Y. pseudotuberculosis}

Although Y. pestis and Y.pseudotuberculosis are nearly identical at the genomic level, they cause very different diseases. Population genetics analysis revealed that $Y$. pestis evolved from $Y$. pseudotuberculosis no earlier than 26,000 years ago (Achtman et al., 1999; Morelli et al., 2010). As a recently emerged pathogen, $Y$. pestis shares some virulence determinants with its ancestor, Y. pseudotuberculosis, a gastrointestinal pathogen.

Type III secretion system (T3SS) is a well-known anti-host system responsible for the virulence of many pathogenic bacteria. T3SS in $Y$. pestis is composed of a secretion machinery, a set of translocation proteins, a control system, and six Yop effector proteins (Cornelis, 2002). Y. pestis injects effectors into the cytosol of eukaryotic cells when docking on the surface of host cells, thereby suppressing phagocytosis and host inflammatory response. T3SS in Y. pestis is encoded by a $70 \mathrm{~kb}$ plasmid, termed as pCD1, which is also found in two other pathogenic Yersinia species, Y. pseudotuberculosis and Y. enterocolitica. The loss of T3SS is sufficient to render $Y$. pestis completely avirulent, even when the bacteria are directly introduced into the bloodstream (Viboud and Bliska, 2005).

Escaping from macrophages at the early stage of infection is a vital step for $Y$. pestis, a facultatively intracellular pathogen. Interestingly, the ability to survive and replicate in macrophages is conserved in Y. pestis and Y. pseudotuberculosis (Pujol and Bliska, 2003). RipABC, MgtCB, Ugd, Yfe, and Feo have been shown to be required for the replication of $Y$. pestis in macrophages (Zhou and Yang, 2009). 
Iron is well established as an essential nutrient chelated by mammalian proteins, lessening its availability to invading pathogens. Iron acquisition is critical to the survival of pathogenic bacteria during infection. Several iron acquisition systems have been characterized or annotated in Y. pestis, and at least two (Ybt and Yfe) of them have been proven essential to its full virulence (Gao et al., 2008; Sebbane et al., 2010). Ybt, also known as the high-pathogenicity island in the $102 \mathrm{~kb}$ pgm locus (Fetherston and Perry, 1994), is necessary for iron acquisition at the flea bite site and in the lymphatic system. On the other hand, Yfe is likely to exert effects in the later stages of the disease, i.e., blood-borne systemic dissemination (Gao et al., 2008).

\section{Y. pestis-SPECIFIC VIRULENCE-ASSOCIATED GENES}

$Y$. pestis and $Y$. pseudotuberculosis have considerably similar genome contents. For instance, $75 \%$ of the annotated genes in the IP32953 chromosome of Y. pseudotuberculosis have no less than $97 \%$ identity (nucleotide level) to their homologs in $Y$. pestis (Chain et al., 2004). Horizontal gene transfer (HGT), also referred to as lateral gene transfer, is a major force in the evolutionary scenarios of bacteria (Gogarten et al., 2002). Some Y. pestis-specific genes acquired or lost by HGT are responsible for its unique virulence.

One major step in the speciation of $Y$. pestis is the acquisition of two unique virulence plasmids, pPCP1, and pMT1 (Achtman et al., 2004). Plasmid pPCP1 encodes the plasminogen activator (Pla), which is essential to both bubonic and primary pneumonic plagues (but not the primary and secondary septicemic forms) (Sodeinde and Goguen, 1988; Sodeinde et al., 1992), promotes $Y$. pestis dissemination from peripheral infection routes, and is responsible for the flea-borne transmission of the plague (Sebbane et al., 2006a; Lathem et al., 2007).

Plasmid pMT1 encodes an F1 antigen and murine toxin (Ymt). $Y$. pestis expresses a unique capsule-like $\mathrm{F} 1$ antigen at $37^{\circ} \mathrm{C}$ but not at $26^{\circ} \mathrm{C}$, which are the human and flea body temperatures, respectively. F1 antigen provides $Y$. pestis the ability to block phagocytosis through a mechanism different from those of T3SS and pH 6 antigen (Du et al., 2002). Ymt shows phospholipase D activity but does not play a direct role in mouse plague infection (Hinnebusch et al., 2000). Further studies have revealed that the phospholipase D activity of Ymt is required for the survival of $Y$. pestis in the midgut of fleas by affording protection against a cytotoxic digestion product of blood plasma in the flea gut. By enabling the colonization of the flea midgut, Ymt acquisition may facilitate the transition of $Y$. pestis to an obligate flea-borne transmission style (Hinnebusch et al., 2002).

Various plasmids have been identified in different $Y$. pestis strains, such as 6-kb pYC (Dong et al., 2000), 22-kb pCRY (Song et al., 2004), and the $183 \mathrm{~kb}$ multiple antibiotic resistance plasmid pIP1202 (Welch et al., 2007). However, none of them have been proven to be involved in the virulence of $Y$. pestis.

A microarray-based genomic comparison of $Y$. pestis has identified an Orientalis strain-specific chromosomal region termed as DFR13 (Zhou et al., 2004b). This region encodes a filamentous prophage (YpfF) (Gonzalez et al., 2002), which is also called CUS-2(Cathelyn et al., 2007; Derbise et al., 2007). Deletion of YpfF from the $Y$. pestis genome does not affect its ability to colonize and block the flea proventriculus, but results in an alteration of $Y$. pestis pathogenicity in mice. Although $\mathrm{YpfF}$ is stably integrated into the genome of biovar Orientalis strains of $Y$. pestis, it forms an unstable episome in other Y. pestis biovars (Derbise et al., 2007). Screening YpfF in a larger collection of $Y$. pestis strains has confirmed this conclusion (Li et al., 2008a).

The identification of virulence genes other than the aforementioned ones from $Y$. pestis-specific genes is laborious and difficult. A detailed in silico comparative analysis of Y. pseudotuberculosis and $Y$. pestis has revealed only eight chromosomal loci (six regions and two genes) specific to Y. pestis. Signatures of integration by site-specific or homologous recombination are identified for most of them, suggesting they are imported by HGT. Intriguingly, depletion mutants of these loci exhibit no defect during growth in vitro. All depletants are also fully virulent and do not show detectable differences in the capacity to block the flea midgut. They also do not produce stable and persistent infection through subcutaneous or respiratory (aerosol) routes of infection. These results suggest that chromosomal acquisition may not have been of major importance in the remarkable change in life cycle that has accompanied the emergence of $Y$. pestis (Derbise et al., 2010).

\section{GENOME DECAY}

Genome decay (gene inactivation and gene loss), a common theme in the evolution of bacterial pathogens, occurs when a gene (or gene cluster) is no longer used by the microbe or when a microbe attempts to adapt to a new ecological niche (Cerdeno-Tarraga et al., 2004). Loss of the unnecessary genetic elements offers the pathogens better fitness when new niches are encountered.

A major finding in the first published $Y$. pestis genome is that CO92 harbors a large number of insertion sequences (IS) that comprise $3.7 \%$ of the genome, which is far more than in most other known bacteria. IS interruption and frameshift mutations have shaped 149 pseudogenes in the CO92 genome (Parkhill et al., 2001). A brief comparison of the CO92 and Y. pseudotuberculosis IP32953 genomes identified 317 genes absent from Y. pestis, which means that a large proportion of $Y$. pseudotuberculosis genes $(13 \%)$ are no longer functional (inactivated or absent) in Y. pestis (Chain et al., 2004). The accumulation of pseudogenes may promote the speciation and microevolution of $Y$. pestis (Tong et al., 2005).

A comparison of the genomes of $Y$. pestis and $Y$. pseudotuberculosis has identified nine loci (five genomic regions and four individual genes), which are supposed to be present in $Y$. pseudotuberculosis and absent in $Y$. pestis. The deletion of $\mathrm{R} 1$, a region predicted to encode the methionine salvage pathway, altered the pathogenicity of mutant Y. pseudotuberculosis. Interestingly, R1 is present and conserved in two $Y$. pestis strains on the ancient lineage, implying R1 loss as an early step in Y. pestis microevolution. Region R3 has also been proven to be sequentially lost in the Y. pestis genomes (Pouillot et al., 2008). These gene-loss events reflect past decays of the $Y$. pestis genome, which is still actively evolving as an obligate intercellular pathogen with minimized genome (Moran, 2002; Ochman, 2005). 
For example, yadA and inv genes respectively encode for the major adhesin and invasin in $Y$. pseudotuberculosis, which are essential for this enteropathogen to adhere onto host intestinal surfaces and invade epithelial cells (El Tahir and Skurnik, 2001; Superti et al., 2005). Both genes are inactivated in Y. pestis (Rosqvist et al., 1988; Simonet et al., 1996; Parkhill et al., 2001). Another important pseudogene in $Y$. pestis is ureD, which is inactivated by a premature stop codon, resulting in a malfunctioned ure operon (Sebbane et al., 2001). Urease is necessary for the oral transmission of Y. pseudotuberculosis; however, it is redundant in the flea-host-flea cycle of $Y$. pestis and may have been abandoned during its process of host adaption (Chen et al., 2010).

$Y$. pestis expresses rough LPS lacking the $\mathrm{O}$ antigen because of the inactivation of five genes in the O-antigen gene cluster (Parkhill et al., 2001). The loss of O-antigen and expression of rough LPS are believed to facilitate the Pla functions and invasiveness of Y. pestis (Kukkonen et al., 2004).

The transcriptional regulator RcsA is known to inhibit Yersinia biofilm formation. $r c s A$ is functional in Y. pseudotuberculosis but is a pseudogene in $Y$. pestis. Substitution with the functional homolog from $Y$. pseudotuberculosis into $Y$. pestis abolishes its biofilm formation in fleas (Sun et al., 2008). The pseudogenization of $\operatorname{rcs} A$ is a clear case of positive selection during the evolution of $Y$. pestis.

\section{METABOLIC ENZYMES AND PATHWAYS}

Ten years ago, Dr. Brubaker (1991) summarized the metabolic differences among Y. pestis, Y. pseudotuberculosis, and Y. enterocolitca, including the following metabolic defects in Y. pestis: biosyntheses of methionine, phenylalanine, threonine-glycine, and isoleucinevaline. The fermentation of rhamnose and melibiose is observed only in the biovar Microtus strains of $Y$. pestis, the closest lineage to Y. pseudotuberculosis that can ferment these two sugars (Zhou et al., 2004c; Zhou and Yang, 2009; Morelli et al., 2010). Both $Y$. pestis and Y. pseudotuberculosis cannot ferment sucrose and sorbitol compared with Y. enterocolitica, and all these three Yersinia species cannot ferment cellobiose (Brubaker, 1991). Y. pestis cannot produce aspartase (AspA), glucose-6-phosphate dehydrogenase, and urease; only Y. enterocolitica can synthesize ornithine decarboxylase; and only $Y$. pestis possesses constitutive glyoxylate bypass. In addition, among the three species, only $Y$. pestis is unable to assimilate low levels of $\mathrm{NH}_{4}$ at $26^{\circ} \mathrm{C}$ (Brubaker, 1991). The genome sequences of Yersinia are rapidly increasing; thus, a timely comparison of all metabolic differences among Y. pestis and $Y$. pseudotuberculosis can promote hypothesis-driven research on the evolution and pathogenesis of Y. pestis.

AspA catalyzes the deamination of L-aspartate to form fumarate, a component of tricarboxylic acid. Typical Y. pestis strains lack AspA activity, resulting in the excretion of L-aspartic acid at the expense of exogenous L-glutamic acid during expression of the low-calcium response. The accumulation of Lglutamate in vivo may radically alter the equilibrium of host amino acid pools, thereby contributing to enhanced lethality (Brubaker, 2007). Genomic analysis revealed a nonsynonymous substitution in codon 363 of aspA (valine to leucine) in typical Y. pestis isolates such as KIM and CO92 (Chain et al., 2004). Additional studies have revealed that this mutation (a replacement of the aliphatic amino acid leucine for another, valine, in AspA of $Y$. pestis) is not conservative. $K_{\text {cat }} / K_{\mathrm{m}}$ of AspALeu363 in KIM is 500-fold lower than that of AspA-Val363 in Y. pseudotuberculosis (Viola et al., 2008). Screening AspA in more $Y$. pestis strains indicate that certain enzootic (pestoides) isolates of $Y$. pestis possess wild-type (WT) AspA. Additional variants of AspA (Phe363 and Ser363) are also found in other pestoides strains. All tested enzootic isolates produce biologically active AspA, although its specific activity exhibits significant variation depending on the codon 363 mutations (Bearden et al., 2009). The decreased activities of AspA in enzootic isolates may account for their attenuated virulence in mammals other than mice, and the functional AspA may serve as a biomarker for the avirulence of $Y$. pestis in humans (Bearden and Brubaker, 2010).

For a complete understanding of metabolic pathways and virulence, some works have been conducted based on bioinformatical, mathmatical, or experimental analyses (Navid and Almaas, 2009; Charusanti et al., 2011; Lv and Henderson, 2011; Navid, 2011), showing that $Y$. pestis genome-scale metabolic reconstruction can identify the metabolic weaknesses for a rational design of therapeutic agents. A combined metabolomic-genetic approach has demonstrated that virulence-associated secondary metabolite systems may shape bacterial primary metabolism independently of substrate consumption (Lv and Henderson, 2011).

As aforementioned, genomic analysis has revealed various mechanisms involved in the virulence of $Y$. pestis, including HGT, adaptive genome decay, and alterations in certain proteins and/or enzymes, which are far more complicated than expected. The influx of bacterial genome data have challenged the simplistic views that bacterial pathogens can be portrayed by identifying their virulence factors and that pathogens always evolve from non-pathogens by acquiring virulence genes by HGT. An "ecoevo" view of bacterial pathogenomics is very useful in understanding the evolution of complex virulence systems by taking the interaction of pathogens and hosts into account (Pallen and Wren, 2007).

\section{TRANSCRIPTOMICS FOR FINDING EVIDENCE OF $Y$. pestis VIRULENCE}

Bacteria are highly adaptive organisms that inhabit a broad range of ecological niches and constantly face variable environmental conditions. Thus, a pathogenic lifestyle requires strict control of both virulence gene expression and general stress responses. Comprehensive transcriptomics analysis benefits our understanding of the molecular determinants of bacterial pathogenesis and cellular regulatory circuits. These studies have refined the co-regulated genes, and have provided insight into the possible functions of uncharacterized genes and regulatory elements of $Y$. pestis (such as operons and DNA regulatory motifs). This kind of analysis provides an opportunity to gain a global view of the environmental modulation of gene expression patterns in $Y$. pestis, which is also very useful for the further genetic dissection of Yersinia pathogenicity.

\section{DNA MICROARRAY-BASED TRANSCRIPTOMICS}

The DNA microarray is an ideal tool for the genome-wide analysis of gene regulation at the transcriptional level. Comprehensive 
analysis of large sets of microarray expression data is useful for dissecting bacterial adaptation to various environments and for understanding bacterial gene transcriptional regulation (Goodman and Lory, 2004; Thompson et al., 2006). Environmental modulation of gene expression in $Y$. pestis is critical to its lifestyle and pathogenesis. To provide a comprehensive view of the environmental modulation of global gene expression in $Y$. pestis, we have analyzed the gene expression profiles of multiple stress conditions (Han et al., 2004, 2005a,b; Motin et al., 2004; Qiu et al., 2005, 2006; Zhou et al., 2005, 2006a,c), including temperature alteration, increased osmolarity, ion deficiency, antibiotic treatment, oxidative, and acidic stresses, antibacterial peptide treatment, as well as nutrition limitation.

\section{Transcriptional regulation of virulence genes}

The stress conditions used in array experiments are hypothesized to be encountered by $Y$. pestis during its infection and life cycle. Identification of the expression patterns of virulence genes within a wide range of environmental changes provides a reference for screening uncharacterized genes that show the same differential gene expression under the same stressful conditions.

The transmission and infection of $Y$. pestis can be roughly divided into distinct stages: maintenance in fleas, adhesion onto host surface, invasion into epithelial or endothelial cells, intracellular growth, antiphagocytosis, and extracellular proliferation. $Y$. pestis possesses a set of virulence determinants that promote infection in mammalian hosts and/or transmission by flea vectors. Different virulence genes have also been proven or proposed to be involved in different infection stages as reviewed (Perry and Fetherston, 1997; Zhou et al., 2006b). The array data supported the notion that $Y$. pestis has evolved the ability to regulate coordinately a large set of genes to survive an organized wide range of environmental perturbations (Han et al., 2007).

As previously described, the expression profiles of $Y$. pestis show that almost all the putative virulence genes of $Y$. pestis are differentially regulated upon temperature alteration (Han et al., 2004; Motin et al., 2004; Han et al., 2005b). Differential gene expression at these two temperatures is believed to allow the bacterium to colonize its host efficiently, leading to its pathogenesis. The known $Y$. pestis virulence genes also respond to many other environmental stresses. For example, the hemin storage locus hmsHFRS (Pendrak and Perry, 1991) is repressed by temperature upshift, high osmolarity, nutrition limitation, and streptomycin treatment. The $y m t$ gene encoding Yersinia murine toxin (Lindler et al., 1998) is also regulated by temperature upshift and streptomycin treatment.

$Y$. pestis synthesizes several antiphagocytic factors, including F1 capsular antigen (Du et al., 2002), pH6 antigen (Huang and Lindler, 2004), and Yersinia outer proteins (Yops) (Cornelis et al., 1998). The expression of Yops is regulated by temperature alteration, increased osmolarity, and nutrition deficiency under normal $\mathrm{Ca}^{2+}$ conditions. These data suggest that the lowcalcium response of T3SS appears to be triggered at the mRNA level by other environmental cues in addition to temperature upshift and $\mathrm{Ca}^{2+}$ limitation. F1 capsular antigen is expressed much more at $37^{\circ} \mathrm{C}$ than at $26^{\circ} \mathrm{C}$ (Simpson et al., 1990). pH6 antigen (PsaA), encoded by the chromosomal psaA gene, expresses in vitro between pH 5 and 6.7 from 35 to $41^{\circ} \mathrm{C}$ (Zav'yalov et al., 1996), or when bacteria live within phagocytic phagolysomes (Makoveichuk et al., 2003). The psaEFABC operon encodes a chaperone/usher pathway involved in the secretion and assembly of pH6 antigen as a polymer (fimbriae) on the surface of $Y$. pestis in macrophages (Zav'yalov et al., 1996; Payne et al., 1998). PsaE is believed to be a positive regulator of the $p s a A B C$ locus and is required for the maximal expression of pH6 antigen (Lindler et al., 1990). A study showed that the psaEFABC locus is regulated by RovA (Cathelyn et al., 2006). The microarray data show that the F1 operon is upregulated upon temperature upshift, low $\mathrm{pH}$, oxidative stress, low $\mathrm{Mg}^{2+}$, and nutrition deficiency. The psaEFABC locus is induced by temperature alteration, acid stress, low $\mathrm{Mg}^{2+}$, nutrition limitation, high salinity, and hyperosmotic stress. The synergistic operation of complicated microenvironments within mammalian hosts can be reasonably assumed to account for the full expression of these two loci ( $p s a A B C$ and psaEFABC).

To evaluate any specific contribution of one gene to host cell interaction, many efforts to measure the gene expression of $Y$. pestis systematically under relevant infection conditions have also been performed. $Y$. pestis transcriptomes in vivo have been analyzed in different infection models; different sets of differentially expressed genes represent distinct niches inhabited by $Y$. pestis. Two research groups have provided the in vivo transcriptome analysis of $Y$. pestis by establishing a primary pneumonic plague in mice (Lathem et al., 2005; Lawson et al., 2006). When compared with in vitro growth at $37^{\circ} \mathrm{C}$, the plasmid pCD1encoded T3SS and many genes located in the pigmentation ( $\mathrm{pgm}$ ) locus of the chromosome are significantly induced in the lungs of infected mice. Two other virulence determinants, Pla and $\mathrm{pH} 6$ antigen, are downregulated in vivo after $48 \mathrm{~h}$ of intranasal infection. In addition to the virulence determinants, genes involved in the detoxification of reactive oxygen species and multiple genes involved in the stress response are differentially regulated in vivo. The transcriptional profile of $Y$. pestis during the mouse bubonic plague shows similarities to and differences from that of $Y$. pestis during the primary pneumonic plague (Sebbane et al., 2006b). The $Y$. pestis T3SS and F1 capsule genes are highly expressed in both pneumonic and bubonic plague mouse models, whereas $\mathrm{Pla}$ is not regulated in the lymph node (bubonic plague). The upregulation of genes required for iron acquisition and for resistance to nitrosative stress suggests that iron deprivation and NO-induced stress are more severe in the rat bubo than in the mouse lung. Chauvaux et al. (2007) have analyzed the expression profile of $Y$. pestis CO92 incubated in decomplemented human plasma mimicking the septicaemic plague. Dozens of genes for iron acquisition or storage systems are particularly induced in the human plasma. pH6 antigen and four other fimbrial-like proteins encoding genes are also regulated in vivo. The ability of $Y$. pestis to survive inside macrophages has been established to be critical during the early stages of plague pathogenesis (Straley and Harmon, 1984; Charnetzky and Shuford, 1985). A number of stress-response genes, including those involved in the detoxification of reactive oxygen species, as well as several metabolic genes involved in macromolecule synthesis, are found strongly induced in intracellular $Y$. pestis. This finding is consistent with 
the presence of oxidative stress and nutrient starvation inside Yersinia-containing vacuoles (Fukuto et al., 2010). In the fleaborne transmission model, several genes involved in resistance to innate immunity ( $p h o P$ and $m g t C$ ) and pathogenicity ( $y a d B C$ and $P l a)$ in mammals are found to be upregulated in the flea proventriculus (Vadyvaloo et al., 2010). The upregulation of these genes may enhance the survival and dissemination of $Y$. pestis in the early stage after transmission to the mammalian host.

\section{Clustering analysis and functional classification of co-expressed genes}

Clustering microarray expression data can be viewed as a data reduction process wherein observations of gene expression in each cluster can be over-represented. This process provides considerable insight into functional classes of co-expressed genes because the genes that are functionally related should be coregulated and, consequently, should show similar expression profiles. Thus, clustering genes with similar expression patterns can potentially be utilized to predict the functions of gene products with unknown functions, as well as to identify sets of genes that are co-expressed and may play the same roles in different cell cycles (Eisen et al., 1998; Herrero and Dopazo, 2002). Clustering analysis of the entire microarray dataset was performed, and the four distinct clusters of co-expressed genes such as clusters I-IV are identified. The possible roles of uncharacterized genes may be inferred by referencing other members in each cluster. Cluster I consists of more than 70 genes, and most of which are functionally related to biosynthesis of ribosomal proteins. The ribosome is the site of protein synthesis and it determines the capacity of the cell to synthesize proteins, thereby determining the growth rate of bacteria. Most members of Cluster I are downregulated in response to a temperature shift from 26 to $37^{\circ} \mathrm{C}$, high osmolarity, $\mathrm{Mg}^{2+}$ limitation, nutrition deficiency, and antibiotic treatment. Thus, Y. pestis appears to slow its growth rate under these conditions. Cluster II contains dozens of genes involved in iron/heme assimilation. Noticeably, almost all genes in this cluster are upregulated in response to iron scavenging in wild type (WT) strains, and to iron excess in ferric uptake regulator (fur) mutant grown at 26 or $37^{\circ} \mathrm{C}$. Cluster III contains members of the cys regulon, including tauABCD, ssuEADCB, cysPUWAM, and sbp1. These genes are regulated by most of the environmental stresses under study. Thus, sulfur metabolism may play important roles in the adaptation of $Y$. pestis to various environmental perturbations. Cluster IV includes $s d h C D A B$ and $s u c A B C D$ (involved in the tricarboxylic acid cycle), which have expression patterns similar to those of nuoA-N and cyoABCDE (involved in aerobic respiration). The microarray data show that these energy metabolism-related genes are downregulated upon heat shock, high osmolarity, $\mathrm{Mg}^{2+}$ limitation, and streptomycin treatment, but are upregulated upon chloramphenicol treatment. These results indicate that a general retardation of energy generation in $Y$. pestis may occur in response to such suboptimal growth conditions.

\section{cDNA LIBRARY- OR RNA SEOUENCING (RNA-SEQ)-BASED TRANSCRIPTIONAL ANALYSIS OF $\boldsymbol{Y}$. pestis REGULATORY ncRNAs}

Traditionally, studies on virulence-related regulations have been focused on the transcription factors that switch on or off relevant sets of genes in response to environmental cues. By contrast, the roles of small (noncoding) RNAs (sRNAs) in pathogenesis have only begun to be addressed. sRNAs are crucial regulators that enable the cell to modulate a wide range of physiological responses via various mechanisms. They are usually untranslated and 50-500 nucleotides in length. Most sRNAs interact with specific messenger RNAs (mRNAs) or protein targets by the trans-acting mode or structure-based interactions. The interaction results in the modulation of mRNA stability, translation, and protein activity (Narberhaus and Vogel, 2009; Waters and Storz, 2009). Hfq is postulated to be an RNA-binding protein, working in conjunction with many sRNAs. Interestingly, Hfqmediated regulation seems to be implicated in the complete infective life cycle of the plague. Hfq is also shown to be a key regulator involved in $Y$. pestis stress resistance, intracellular survival, and pathogenesis (Geng et al., 2009), as well as a requirement for biofilm-mediated gut blockage in fleas by modulating the intracelullar levels of c-di-GMP (Bellows et al., 2012; Rempe et al., 2012). Hfq is now accepted to function by stabilizing and facilitating base-pairing between sRNAs and their cognate mRNAs (Vogel and Luisi, 2011). Therefore, Hfq acts by controlling the expression of many virulence-, biofilm-, and stress-associated genes, probably in conjunction with sRNAs. Attempts on the global screening of Hfq-dependent sRNAs have been made in Escherichia coli (Zhang et al., 2003). SgrS, an Hfq-dependent sRNA, is found to be induced under glucose-phosphate stress conditions and responsible for the destabilization of $p t s G$ mRNA, which encodes the major glucose transporter of the phosphoenolpyruvate phosphotransferase system (Wadler and Vanderpool, 2007). SgrS is a dual-function sRNA with base-pairing and mRNA functions. A truncated SgrS homolog found in Y. pestis is still functional under glucose-phosphate stress (Horler and Vanderpool, 2009; Wadler and Vanderpool, 2009); however, its direct relevance with $Y$. pestis virulence is unknown. Hfq-dependent sRNAs reportedly contribute to $Y$. pestis virulence (Koo et al., 2011). Hence, the mechanism of known or newly discovered Hfq-dependent sRNAs involved in $Y$. pestis infection must be elucidated.

RNA-seq technology can enable the deep sequencing of cDNA generated from RNA preparations. Compared with tiling arrays, RNA-seq provides a better signal-to-noise ratio because of a reduced background and a higher dynamic range (Guell et al., 2011). Using this technology, 150 novel sRNAs have been recently identified in Y. pesudotuboculosis, the most closely related species to $Y$. pestis. One sRNA chosen for preliminary evaluation is found to play certain roles in the pneumonic plague (Koo et al., 2011). In a recently published study of our group, we used RNomics to find 43 highly abundant sRNAs in $Y$. pestis under multiple growth conditions (Qu et al., 2012). These highly expressed sRNAs under simulated conditions may play important roles in $Y$. pestis adaptability and pathogenesis. Analysis of the expression patterns of 29 candidate sRNAs shows that 24 sRNAs are highly abundant in $Y$. pestis upon entry into the stationary growth phase.

\section{REGULATION OF THE EXPRESSION OF VIRULENCE DETERMINANTS}

The expression of virulence determinants, which allows $Y$. pestis to multiply on and within host cells and tissues, is strictly and 
coordinately regulated by various regulators. As a member of the MarR/SlyA family of transcriptional regulators that control the virulence of multiple bacterial pathogens (Ellison and Miller, 2006), RovA is required for the virulence of all three pathogenic Yersinia species (Y. pestis, Y. pseudotuberculosis, and Y. enterocolitica) through the regulation of various virulence loci (Revell and Miller, 2000; Nagel et al., 2001; Dube et al., 2003; Ellison et al., 2004; Cathelyn et al., 2006). In Y. pseudotuberculosis and Y. enterocolitica, RovA stimulates the transcription of inv, which encodes an invasin that mediates translocation across the intestinal epithelium (Revell and Miller, 2000; Nagel et al., 2001; Ellison et al., 2004; Heroven et al., 2004). The rovA null mutant of $Y$. pestis is much more attenuated after subcutaneous inoculation than after an intranasal or intraperitoneal route, which indicates the more important role of RovA in subcutaneous infection than in pneumonic or systemic ones (Cathelyn et al., 2006). In $Y$. pestis, RovA stimulates the transcription of the $p s a E F, p s a A B C$, and CUS-2 prophage loci (Cathelyn et al., 2006). pH6 antigen encoded by $p s a A B C$ acts as an antiphagocytic factor (Huang and Lindler, 2004) and plays a more important role in bubonic plague than in the pneumonic and septicemic forms, closely mimicking the role of RovA (Cathelyn et al., 2006). The RovA regulator still plays critical roles in the construction and functioning of the bacterial membrane, indicating the regulatory functions of RovA in antibiotic resistance and environmental adaptation (Yang et al., 2010).

PhoP and PhoQ constitute a classic regulatory two-component system (Groisman, 2001). The sensor protein PhoQ responds to low environmental $\mathrm{Mg}^{2+}$, acidic $\mathrm{pH}$, and host-secreted antimicrobial peptides, and then phosphorylates the response regulator PhoP. As a transcription factor, phosphorylated PhoP either activates or represses its target genes by binding with their promoter-proximal DNA regions. A phoP null mutant of $Y$. pestis shows reduced ability to survive in macrophages and human neutrophils, as well as under in vitro conditions of low $\mathrm{pH}$, oxidative stress, high osmolarity, and antimicrobial peptides (Oyston et al., 2000; Hitchen et al., 2002; O'Loughlin et al., 2010). This mutant is slightly attenuated in mice (Oyston et al., 2000); however, the $\mathrm{LD}_{50}$ of $Y$. pestis $\Delta$ phoP mutant does not differ from the WT strain for either the bubonic or pneumonic murine models of infection (Bozue et al., 2011). As a global regulator, PhoP controls a very complex regulatory cascade in Y. pestis (Li et al., 2008b; Perez and Groisman, 2009; Perez et al., 2009). The PhoP regulons in $Y$. pestis and Salmonella enterica considerably differ in terms of the functional changes in PhoP itself, as well as in the architecture of PhoP-dependent promoters. This difference allows the PhoP regulators to incorporate newly acquired genes into the ancestral regulatory circuits in these two bacteria (Perez and Groisman, 2009; Perez et al., 2009). The proven direct PhoP targets in Y. pestis include several genes that function in detoxification, protection against DNA damage, resistance to antimicrobial peptides, and adaptation to magnesium limitation ( $\mathrm{Li}$ et al., 2008b). In particular, the $m g t C B$ and $u d g$ loci that encode an $\mathrm{Mg}^{2+}$ transport system and a UDP-glucuronate decarboxylase for LPS modification, respectively, are required for the replication of $Y$. pestis in macrophages (Grabenstein et al., 2006). These PhoP-dependent mechanisms used by $Y$. pestis contribute to the intracellular growth of this pathogen.

The cyclic AMP receptor protein (CRP) controls the transcription of more than 100 bacterial genes/operons (Zheng et al., 2004). CRP is active only in the presence of cyclic AMP that behaves as a classic small-molecule inducer. The crp deletion leads to a huge attenuation of virulence of $Y$. pestis after subcutaneous infection of mice (Zhan et al., 2008). The expression of Pla, pesticin (Pst), and type III YOP secretion components depends on CRP in Y. pestis (Lee et al., 2007; Zhan et al., 2008, 2009). Specifically, CRP directly stimulates the expression of Pla (Kim et al., 2007; Lee et al., 2007; Zhan et al., 2008), a virulent factor essential to the bubonic and primary pneumonic plagues (Sodeinde et al., 1992; Lathem et al., 2007). Given that Pla specifically promotes $Y$. pestis dissemination from peripheral infection routes, the defective expression of Pla in the crp mutant significantly contributes to the huge loss of virulence of this mutant strain after subcutaneous infection (Zhan et al., 2008).

Fur is a predominant iron-regulating system in bacteria (Escolar et al., 1999). Fur directly controls almost all iron assimilation functions and a variety of genes involved in various non-iron functions, and thus governs a complex regulatory cascade in Y. pestis (Zhou and Yang, 2006; Gao et al., 2008). A variety of iron acquisition systems have been characterized in Y. pestis, and at least two (Ybt and Yfe) of them are proved to be required for full virulence (Bearden et al., 1998; Bearden and Perry, 1999). The $y b t$ locus is transcribed into four operons ( $f y u A$, irp2-irp1-ybtUTE, $y b t A$, and $y b t P Q X S$ ) (Carniel, 1999, 2001). Fur repressed the whole $y b t$ locus in response to excess extracellular iron. In addition to Fur, YbtA has been shown to be another transcriptional regulator that functions as an activator of irp2irp1-ybtUTE, $y b t P Q X S$, and $f y u A$, and as a repressor of its own transcription (Fetherston et al., 1996; Anisimov et al., 2005). Fur and YbtA share all four operons within the $y b t$ locus as the direct targets at the transcriptional level (Gao et al., 2008).

The above regulators and their target virulence genes constitute a prototype of virulence gene regulatory network. Most of the abovementioned virulence factors are acquired through HGT. Thus, the newly acquired virulence genes, which are expressed at high levels during specific stages of infection, have been integrated into the host gene regulatory network controlled by the host regulators.

For many Gram-negative pathogens, hexa-acylated LPS can efficiently activate toll like receptor 4 (TLR4) signaling and further stimulate the host innate immune response. Both $Y$. pestis and $Y$. pseudotuberculosis do not carry the $l p x L$ gene, which encodes an enzyme that transfers the secondary laurate chain to lipid $\mathrm{A}$ at $37^{\circ} \mathrm{C}$ and results in a tetra-acylated LPS with poor binding ability to TLR4, whereas Y. enterocolitica carries LpxL (HtrB) (Perez-Gutierrez et al., 2010). The remaining $l p x M$ and $l p x P$ can make a hexa-acylated lipid A in Y. pestis at ambient temperature, but at $37^{\circ} \mathrm{C}$, plague bacteria produce mainly tetra-acylated LPS. Thus, a differential acylation of the lipid A in Y. pestis is a temperature-regulated process (Kawahara et al., 2002; Telepnev et al., 2009). Introducing $l_{p} x L$ into $Y$. pestis results in the formation of hexa-acylated lipid $\mathrm{A}$ at $37^{\circ} \mathrm{C}$ and ablating virulence in mice (Montminy et al., 2006). Clearly, the loss of $l p x L$ plays a 
vital role in the immune evasion of $Y$. pestis, and an $l p x L$ knock-in mutant of $Y$. pestis KIM strain has been proposed as a novel live vaccine against plagues (Sun et al., 2011).

\section{HOST TRANSCRIPTOMIC RESPONSE TO $Y$. pestis INFECTION}

DNA microarray technologies are widely used to investigate not only the bacterial transcriptome under various stimulant conditions, but also the host transcriptomic responses to microbial infections. Y. pestis infection-induced host transcriptomic responses have been investigated in both bubonic and pneumonic plague animal models, as well as in different types of cultivated cells. The combination of results from host transcriptomic responses, pathological studies on the tissues of infected animals, and pathogenic mechanisms of specific virulent factors of this pathogen has significantly improved our understanding of the crosstalk between $Y$. pestis and a host.

\section{Ex vivo TRANSCRIPTIONAL RESPONSES IN INFECTED CELL CULTURES}

$Y$. pestis can survive and replicate in macrophages by inhibiting the acidification of phagosomes in vitro and in vivo on bubonic mice model, but are killed in neutrophils (Lukaszewski et al., 2005). Evidence shows that neutrophils restrict the growth of $Y$. pestis, whereas macrophages do not (Lukaszewski et al., 2005). Macrophages actually provide a protected environment for organisms to synthesize their capsular layer and other antiphagocytic mechanisms (Pujol et al., 2009). For a better understanding of the interactions between $Y$. pestis and a host at the early stage of infection, several groups have investigated the transcriptional response of cultivated macrophages or human peripheral blood lymphocytes to $Y$. pestis infection. Subrahmanyam et al. (2001) have applied a cDNA display technique to study mRNA level changes in human neutrophils following bacterial exposure using full virulence $Y$. pestis KIM5, pCD1-defective avirulent $Y$. pestis KIM6, and nonpathogenic E. coli $\mathrm{K} 12$ as the model bacteria. More than 300 genes are observed to be differently expressed in neutrophils exposed to bacteria, including genes of a variety of cytokines, receptors, apoptosis regulating products, and membrane trafficking regulators. A cluster of genes are E. coli and KIM6 responsive but unresponsive in neutrophils exposed to KIM5. For instance, the phagocyte oxidase system generating reactive oxygen is downregulated in KIM-infected neutrophils. By contrast, the free radical scavenging enzyme SOD2 is upregulated by nonpathogenic bacteria. Das et al. (2007) have reported transcriptomic response in cultured human monocytes and lymphocytes exposed to intracellular Y. pestis. Human monocytes are infected with $Y$. pestis bacteria grown at $26^{\circ} \mathrm{C}$, and the extracellular bacteria are killed with gentamicin after $30 \mathrm{~min}$. The ensuing transcriptional changes are largely due to the intercellular bacteria and reflect the host transcriptomic response at the intracellular life cycle of $Y$. pestis. Genes encoding cytokines as well as chemokines, transcription factors, inflammatory, and apoptosis-related genes are significantly changed, indicating that the infection of human monocytes with $Y$. pestis results in a strong inflammatory response (Das et al., 2007). Proinflammatory cytokines and chemokines such as TNF- $\alpha$, macrophage inflammatory protein-1 $\alpha$ (MIP-1 $\alpha)$, MIP- $1 \alpha$, and interleukin-6 (IL6) are upregulated 10-120-fold higher than untreated controls, whereas growth factor- $\beta$ (TGF- $\beta$ ), MIP- $2 \alpha$, and placental growth factor are moderately upregulated (2-15-fold). The induction of the cytokine expression of TNF- $\alpha$, IL-6, and MIP- $1 \alpha$ peaks at $2-4 \mathrm{~h}$ and is eventually downregulated at $8 \mathrm{~h}$, post-infection (p.i.) accompanied with the induction of the anti-inflammatory cytokine IL-10. This finding implies the inhibition of the initial inflammatory response. However, no significant transcriptional change can be detected in infected human lymphocytes, consistent with a previous report that $Y$. pestis preferentially infects monocytes (Marketon et al., 2005). The apoptosis of infected monocytes is inhibited by the downregulation of proapoptotic genes such as peripheral myelin protein 22 (PMP22), caspase-8, and complement component 5 receptor (C5AR), as well as the upregulation of apoptosis inhibitors such as B-cell lymphoma protein $2(\mathrm{Bcl}-2)$. This phenomenon is in contrast to the wellestablished function of YopJ, which has been shown to induce apoptosis in macrophages by blocking mitogen-activated kinase and NF-кB signaling events (Lemaitre et al., 2006; Mukherjee et al., 2006). This contradiction is probably due to the fact that Yops protein cannot be efficiently delivered into the host cells in an intercellular infection experiment. The cellular functions of homeostasis and coagulation are also persistently downregulated after exposure to $Y$. pestis, which favor clot formation and contribute to disseminated intravascular coagulation, a major cause of death from the plague.

To determine the contributions of Yops and T3SS to its pathogenesis, the transcriptional responses of J774A.1 or bone marrowderived macrophages (BMDM) from BALB/c to an infection with $Y$. enterocolitica and various mutants with pYV defections ( $\mathrm{pYV}$ cured strain and Yop mutants including yopJ, yopM, and yopH deletions) are analyzed (Hoffmann et al., 2004). The results show that pYV-cured $Y$. enterocolitica induces a general inflammatory response in infected J774A.1 macrophages, whereas a WT Y. enterocolitica strain induces the expression of genes that have silencing functions in inflammatory responses and can successfully suppress this response. Similar results have been reported in the lymph node from a bubonic plague model, which shows significantly delayed immune response in WT Y. pestis but not in $\mathrm{pYV}^{-}$ mutant (Comer et al., 2010). A comparison of the transcriptional profiles induced by WT Y. enterocolitica and Yops mutants reveals that YopP (YopJ in Y. pestis) mediates the suppression of the inflammatory response; however, neither YopH nor YopM modifies the expression profile on macrophage genes.

A transcriptional response of BMDM from resistant C57BL/6 or susceptible BALB/c mice to infection with $Y$. enterocolitica has been reported. A few genes that activate NK cells, possess antibacterial properties, or are involved in sensing chemokines and LPS are more strongly induced by $\mathrm{C} 57 \mathrm{BL} / 6 \mathrm{BMDM}$ in response to Yersinia sp. infection than BALB/c BMDM (Van Erp et al., 2006). These results indicate that although the host resistance factors modulate a very small portion of transcriptome, the expression of this cluster of genes affects the outcome of the disease.

\section{TRANSCRIPTIONAL RESPONSES TO THE BUBONIC AND PNEUMONIC PLAGUES}

Studies using both bubonic and primary pneumonic plague animal models have revealed that host infections with $Y$. pestis 
show a remarkable biphasic feature in which the infection begins with an early anti-inflammatory phase followed by a proinflammatory phase (Nakajima and Brubaker, 1993; Sebbane et al., 2005; Bergsbaken and Cookson, 2009; Comer et al., 2010). In the bubonic plague, $Y$. pestis rapidly multiplies in draining lymph nodes at the early stage (6-36 h p.i.) with no detectable inflammation. The bacteria then rapidly replicate and disseminate in the blood to colonize the liver, spleen, and lungs. Host immune responses, including phagocyte infiltration, inflammatory cytokine production, and tissue necrosis appears only after $36 \mathrm{~h}$ p.i (Sebbane et al., 2005). Lathem et al. (2005) have also found this biphasic feature in a mouse model of primary pneumonic plague by histopathology inspection. Their results indicate that infection begins with an anti-inflammatory state in the first 24-36 h, and then rapidly progresses to a highly proinflammatory state by $48 \mathrm{~h}$ and death by 3 days. Similarly, robust neutrophil recruitment to the lungs is not observed until $48 \mathrm{~h}$ p.i. in a mouse pneumonic model by Liu et al. (2009). Proinflammatory chemokines are also undetected in bronchoalveolar lavage fluids during this period of infection.

\section{Transcriptional responses to bubonic plague}

The first transcriptomics study on the host response to $Y$. pestis infection was performed in spleens from intraperitoneally (i.p.) infected mice (Rogers et al., 2007). About $48 \mathrm{~h}$ after the mice were i.p. infected with 8 or $257 \mathrm{CFU}$ of $Y$. pestis CO92, the expression of 534 genes were significantly modified in the high-dose infected group, with 384 genes downregulated and 150 genes upregulated. No significant gene expression change was detected in the low-dose infected group, which exhibited no mortality either. Thus, the host immune system successfully controlled the initial spread of $Y$. pestis when the challenge dose was sufficiently low. The altered genes primarily encode proteins in biological processes concerned with immune, cytoskeletal, and cell cycle functions, apoptosis, as well as protein degradation. The upregulated genes associated with immune functions include Src-like adapter, IL-18, TLR8, CD14, and dual specificity phosphatase 1. The downregulated genes contribute to the inhibition of immune response that involves $\mathrm{B}$ cell receptor/co-receptor signaling and subsequent B cell activation, including spleen tyrosine kinase, CD19, complement receptor 2, and E26 avian leukemia oncogene 1 . The expressions of nearly 40 genes related to cytoskeletal remodeling are changed by a $Y$. pestis infection. This finding suggests that Yop effectors (including YopH, YopE, YopT, and YpkA), which can directly target small GTPase family members, exert their virulence function and paralyze the phagocytosis function of host cells.

Comer et al. (2010) have investigated the host response to $Y$. pestis in the lymph nodes of a rat bubonic model. Results show that transcriptomic response in lymph nodes can only be detected after the infection has progressed to septicemic plague ( $60 \mathrm{~h}$ p.i.), which includes the upregulation of some cytokines, chemokines, and other immune response genes. These results are in accordance with the previously reported biphasic features of the plague (Lathem et al., 2005). By contrast, the mutant of Y. pestis lacking the pCD1 plasmid invokes a significant transcriptome response at $36 \mathrm{~h}$. The transcriptomic results are further confirmed by the pathologic observation that the lymph node of an animal infected with $\mathrm{pCD}^{-} Y$. pestis is characterized by sustained recruitment of increasing numbers of neutrophils and the successful clearance of bacteria. This feature highly differs from the WT Y. pestisinfected lymph node. These results show that the pCD1-encoded molecules may induce active immunosuppression at the early stage of the bubonic plague, as supported by a recently published work (Price et al., 2012). Price et al. (2012) have shown that $Y$. pestis rapidly creates a localized, dominant anti-inflammatory state that allows the survival and rapid growth of normally avirulent organisms; however, its progenitor Y. pseudotuberculosis has no such capability. Evidence shows that the T3SS effectors of enteric Yersiniae and $Y$. pestis are not functionally equivalent, or that other virulence factors specific to $Y$. pestis play a role (BaladaLlasat and Mecsas, 2006). Coinfection of WT and $\mathrm{pCD}^{-}$Y. pestis reportedly results in a similar transcriptional response at $36 \mathrm{~h}$ to that aroused by $\mathrm{pCD}^{-}$Y. pestis alone. Thus, WT Y. pestis cannot prevent the immune response aroused by $\mathrm{pCD}^{-} Y$. pestis. Nevertheless, some immune response genes show significant differences between rat infected with $\mathrm{pCD}^{-} Y$. pestis alone and that coinfected with WT Y. pestis. These results imply that a specific part of the immune system can be actively suppressed by pCD1encoded T3SS (Comer et al., 2010). The IL-17 levels in the lymph node and the transcription of the gene for IL-17F are both significantly increased in response to $Y$. pestis infection (Comer et al., 2010). A recent report demonstrates that the increased level of IL17 in the lungs of challenged B cell-deficient mice can improve survival (Lin et al., 2011), suggesting that IL-17-mediated cell immunity plays some roles in host defense against the pneumonic plague.

\section{Transcriptional responses to the pneumonic plague}

Two independent groups have reported the transcriptional responses of different mouse organs (including lungs, spleen, and liver) in an intranasally challenged mouse model of the pneumonic plague (Galindo et al., 2009; Liu et al., 2009). Both studies show a significantly higher number of differentially expressed genes in the lungs at $48 \mathrm{~h}$ than at $12 \mathrm{~h}$ p.i. The small number of changed genes in the lungs from animals exposed to $Y$. pestis is not accompanied by a significant increase in PMNs. Pathological change is also found during the early stage of infection, and strong cytokine production is not detectable until $48 \mathrm{~h}$ p.i. (Liu et al., 2009). CSF-3, MIP-3 $\alpha$, GRO- $\beta$, and IL- $\beta$ are among the most commonly induced cytokines in the lungs at $48 \mathrm{~h}$ p.i. Both CSF- 3 and GRO- $\beta$ target the neutrophils to promote their differentiation, proliferation, and eventual recruitment to the sites of infection. The strong induction of these cytokines reflects a strong immune response at the later stages of the plague. Although significant discrepancies are observed among the mice, cDNA microarray, and data analysis used by the two groups, the majority of the alerted genes well coincided with one another based on a comparison of the published datasets (Galindo et al., 2009).

\section{PROSPECTS AND REMAINING QUESTIONS}

$Y$. pestis is considered to be a clone that branches from its progenitor Y. pseudotuberculosis (Achtman et al., 1999, 2004); however, it is highly virulent compared with its enteropathogenic ancestor. 
The two species share more than $90 \%$ DNA homology and a pCD1/pYV virulence plasmid. Y. pestis has acquired two additional plasmids, namely, pPCP1, and pMT1, facilitating its transmission by fleabite among additional functions. An interesting phenomenon is that the delayed immune response is diminished in a $\mathrm{pCD}^{-}-Y$. pestis infected bubonic rat model, in which a general inflammatory response is evoked in the infected bubo. This finding suggests that T3SS encoded by pCD1/pYV plasmid is responsible for the immune repression induced by $Y$. pestis (Comer et al., 2010). However, no difference is detected in mice subcutaneously challenged with $Y$. pestis or Y. pseudotuberculosis during the progression of the infection to the draining lymph nodes (before 2 days of infection) (Guinet et al., 2008). The subsequent $Y$. pseudotuberculosis infection induces massive PMN influx and the bacterial replication is contained, whereas PMN infiltration is absent in the $Y$. pestis-infected draining lymph nodes, which are typified by an invasion of the tissue by free-floating bacteria. This observation indicates that $Y$. pseudotuberculosis, harboring a pYV plasmid, cannot suppress the host defense response as its descendant, suggesting that chromosomally encoded virulenceassociated factors play roles in this immune suppression. More detailed experiments on host transcriptomics can be designed to investigate the evolution of $Y$. pestis from a moderately virulent enteropathogenic to a highly virulent species.

Next-generation sequencing (NGS) technologies can simultaneously sequence millions of DNA molecules with high throughput efficacy at a lower cost (Wang et al., 2009; Wilhelm and Landry, 2009). NGS-based RNA-seq technologies directly determine the cDNA sequence and generate digital signals that provide highly quantitative and reliable measurements of transcriptional levels (Marioni et al., 2008; Mortazavi et al., 2008). These technologies have been proven to be a powerful alternative to DNAmicroarray-based technology in transcriptomic studies, and can certainly affect this area. RNA-seq is particularly attractive for the quantitative detection of low-abundance transcripts with very high sensitivity, and for the unbiased detection of almost all kinds of transcripts in a given cell type, which is impossible for DNA microarray technology. The application of RNA-seq technology to in vivo and in vitro Y. pestis infection models can enable the collection of delicate and complicated data on host transcriptomic responses.

\section{PROTEOMICS FOR STUDYING Y. pestis VIRULENCE}

Given the numerous genomic and transcriptomic analyses of $Y$. pestis, further research at the protein level (proteomics) can provide more information on the virulence of the pathogen.

Two-dimensional electrophoresis (2-DE) is extensively used as a conventional tool for exploring the virulence factor and the regulatory network involved in the pathogenicity of $Y$. pestis. Pieper et al. (2008) have characterized the periplasmic proteome of $Y$. pestis strain KIM6+ using a differential 2-DE display of proteins isolated from several subcellular fractions. They have found that several periplasmic proteins with unknown functions may play important roles in the $Y$. pestis life cycle. They have also compared the abundance changes at 26 and $37^{\circ} \mathrm{C}$ by analyzing subcellular proteomes, and found that many outer-membrane (OM) proteins, including cell adhesion protein Ail (y1324) and three putative small beta-barrel OM proteins (OMPs) (y1795, y2167, and y4083), are strongly increased at $37^{\circ} \mathrm{C}$. By contrast, the Ail/Lom family protein y1682 (OmpX) is strongly increased at $26^{\circ} \mathrm{C}$ (Pieper et al., 2009). Some type VI secretion system proteins are only identified in membrane fractions of stationaryphase cells grown at $26^{\circ} \mathrm{C}$. Chromy et al. (2005) have used the Ettan 2-D DIGE system to compare the protein expression of Y. pestis at both 26 and $37^{\circ} \mathrm{C}$ with and without $4 \mathrm{mM}$ calcium. They have found the differential expression of several virulenceassociated factors [including catalase-peroxidase (KatY), murine toxin (Ymt), Pla, and F1 capsule antigen (Caf1)], as well as several putative virulence factors and membrane-bound and metabolic proteins. These factors may represent new virulence determinants. Pieper et al. (2010) have compared proteomic changes in three Y. pestis (strain KIM6+) subcellular fractions (soluble periplasmic, cytoplasmic, and mixed membrane fractions) under iron deficiency at two physiologically relevant temperatures $\left(26\right.$ and $\left.37^{\circ} \mathrm{C}\right)$. They have found that five characterized Y. pestis iron/siderophore acquisition systems (Ybt, Yfe, Yfu, Yiu, and $\mathrm{Hmu}$ ) and a putative iron/chelate OM receptor (Y0850) are increased in abundance in iron-starved cells. This result may contribute to the understanding of the important regulatory role of Fur. The iron-sulfur cluster assembly system Suf is functional in $Y$. pestis under iron-limiting conditions. All these comparative studies reveal numerous clues for further functional research on $Y$. pestis; however, the low coverage (usually $20-30 \%$ of all predicted proteins) of 2-DE restricts further analysis. Therefore, different technologies are used in some research. In our laboratory, we have developed a protein microarray consisting of virulence-associated proteins of $Y$. pestis to compare antibody profiles elicited by the WT and quorum-sensing (QS) mutant strain of this bacterium and to define the immunogens affected by QS. The results demonstrate that QS affects the expression of many virulence-associated proteins of $Y$. pestis, including F1, LcrV, KatY, and pH6 antigen (Chen et al., 2006). We have also used this protein microarray to study the host antibody responses to uncover seven $Y$. pestis proteins specifically expressed in vivo during infection ( $\mathrm{Li}$ et al., 2011). Hixson et al. (2006) used the accurate mass and time (AMT) tag mass spectrometry (MS) method and clustering analysis to compare the abundance change in $992 \mathrm{Y}$. pestis proteins under four contrasting growth conditions (26 and $37^{\circ} \mathrm{C}$, with or without $\mathrm{Ca}^{2+}$ ) that mimicked growth states in either a flea vector or mammalian host. They have identified unique biomarkers specifically related to growth conditions. The OMPs are analyzed for a nonpathogenic Y. pestis A1122 strain by liquid chromatography-tandem MS (Jabbour et al., 2010) because OMPs are often associated with virulence in Gram-negative pathogens. However, this study only compares the OMPs between Y. pestis and E. coli, and comparative proteome analysis between pathogenic and non-pathogenic $Y$. pestis can help identify virulence-associated proteins more efficiently.

The recent developments in multidimensional chromatography separation and advanced MS techniques considerably promote research on large-scale proteome profiling (Wall et al., 2000; Washburn et al., 2001; Peng et al., 2003; De Godoy et al., 2006). The implementation of high-profile MS instruments, particularly 
Fourier transform ion cyclone resonance MS and the novel Orbitrap MS, enables the identification of protein mixtures with high throughput and quality (Olsen et al., 2005; Chapman et al., 2006; Heurlier et al., 2006; Zubarev and Mann, 2007). In a typical research on the metal-reducing microorganism Geobacter sulfurreducens using ultra-high-pressure liquid chromatography and MS-based AMT strategy as well as 2-DE, the authors approach about $90 \%$ of the total predicted gene products (Riding et al., 2008). To understand better the physiology and pathogenesis of $Y$. pestis, we have carried out an in-depth proteomic analysis of $Y$. pestis strain 91001 at $26^{\circ} \mathrm{C}$ in a chemically defined medium that mimicks growth states in a flea vector. We have used an advanced LTQ-FT mass spectrometer equipped with a nanospray ion source and an Agilent 1100 Series binary highperformance liquid chromatography system (Zhou et al., 2012). The results demonstrate high coverage [a total of 1926 proteins (13,082 peptides) identified, accounting for 46.50\% (1926/4142) of $Y$. pestis 91001] and high quality [less than $1 \%$ false discovery rate] using this combined strategy. The subsequent transcriptome analysis based on a whole genome DNA microarray of $Y$. pestis defines 1655 genes with $56.65 \%$ coincidence to the proteomic results. Through the comprehensive analysis of the activity of virulent factors involved in the entire life cycle of $Y$. pestis under in vitro flea-simulated conditions, the Hms system and murine toxin (virulence factors involved in $Y$. pestis maintenance in flea) are found to show high expression in our analysis. Some virulence factors are also activated to different extents, such as Pla involved in the adhesion and invasion in mammalian hosts, PhoP/PhoQ two-component system involved in intracellular growth, T3SS, iron acquisition systems (Ybt, Yfe, and Yfu), and Fur involved in extracellular growth and systemic infections. Y. pestis clearly utilizes a variety of survival strategies before invading its hosts.

Proteomics approaches have also been used to study the hostYersinia interactions (Zhang et al., 2005a). Human monocyte U937 cells exposed to Y. pestis, Y. enterocolitica, and Y. pseudotuberculosis were analyzed by $2 \mathrm{DE}$ MS to reveal the host response at the proteomic level (Chromy et al., 2004). Several differentially expressed host proteins involved in protein synthesis, cytoskeletal interactions, immune responses, and apoptosis were identified, suggesting that a specific host protein response profile may be useful for diagnosis of disease without any information of possible pathogens. The same group also quantitatively reveals the protein expression differences from cytoplasmic, nuclear, and membrane fractions of host cells U937 by 2DE DIGE between Y. pestis and Y. pseudotuberculosis challenges (Zhang et al., 2005b). The functions of the differentially expressed proteins can provide insight into the different virulence and pathogenic mechanisms of these two genetically related pathogens.

Ponnusamy et al. (2011) have compared the protein expression differences between intracellularly and extracellularly grown $Y$. pestis using 2DE-MS strategy. The comparison reveals nine proteins that may be involved in stress responses to benefit the intracellular growth of the pathogen in mouse macrophages, including superoxide dismutase-A, inorganic pyrophosphatase, GrcA, DnaK, GsrA, H-NS, UreA, TerD, and TerE.
Our group have used a protein microarray and an enzymelinked immunosorbent spot assay to evaluate both humoral and cellular immune responses to $Y$. pestis infection in long-term recovered plague patients (Li et al., 2012), laying a foundation for new diagnostic development and vaccine design.

\section{INTERACTOMICS FOR REVEALING $\boldsymbol{Y}$. pestis VIRULENCE}

Complicated interactions between the host and pathogen ultimately determine the outcome of a disease. The high-throughput yeast two hybrids $(\mathrm{Y} 2 \mathrm{H})$ system and affinity purification coupled with MS techniques have rapidly progressed in recent years. Accordingly, large-scale investigations on protein-protein interactions (PPIs) by a high-throughput strategy are now widely adopted to investigate the pathogen-host interactions. Despite these advances, we are far from the well understanding of pathogen-host interactomics. The successively published largescale PPIs networks of human and model organisms provide valuable references for investigating protein interaction networks between pathogens and their hosts (Li et al., 2004; Lacount et al., 2005; Rual et al., 2005; Stelzl et al., 2005; Parrish et al., 2007). Viruses are more intensively studied in this aspect than bacterial pathogens partly because of the fact that viruses generally contain relatively small genomes. They also replicate inside the host cells, utilizing the host resources; thus, most of the viral proteins can interact with multiple interaction partners to succeed in this mission. Published virus-host infection networks include hepatitis $\mathrm{C}$ virus, Epstein-Barr virus, and Kaposi sarcoma-associated herpes virus (Uetz et al., 2006; Calderwood et al., 2007; De Chassey et al., 2008). More recently, the interaction networks between human and bacterial pathogens of $Y$. pestis, Bacillus anthracis, and Francisella tularensis have been reported (Dyer et al., 2010). These reports reveal that pathogens tend to interact with hubs (proteins interacting with a large number of partners) and bottlenecks (proteins connecting with many functional modules) in the human PPI network. Distinct pathogens also preferentially interfere with a different spectrum of cellular pathways to facilitate infection and dissemination.

In the study of Yang et al. (2011a), a total of 153 potential virulence-associated proteins of $Y$. pestis are chosen as baits to screen against the human spleen cDNA library using a direct Y2H strategy. More than 200 PPIs between Y. pestis and human proteins were identified, and a Y. pestis-human PPI network was constructed in combination with the published interactions obtained from literature. Results showed that Y. pestis is highly prone to interact with hub and bottleneck proteins essential to normal cellular functions, further supporting previous results that pathogens usually target the central proteins in the human PPI network (Dyer et al., 2008, 2010). The Kyoto Encyclopedia of Genes and Genomes (Aoki-Kinoshita and Kanehisa, 2007) pathways enriched in human proteins targeted by $Y$. pestis proteins highlight a number of important pathways in immune response, including the TLR and MAPK signaling pathways, leukocyte transendoepithelial migrations, focal adhesion, and cytoskeletal regulation. Interference with these pathways by $Y$. pestis helps create a benefit local environment for its replication by modulating the recruitment of leukocytes, inhibiting the phagocytosis of the host phagocytes and interrupting immune signaling events. 
Three interaction networks between human and bacterial pathogens, including $Y$. pestis, have been reported using a random $\mathrm{Y} 2 \mathrm{H}$ approach (Dyer et al., 2010). The human-Y. pestis interaction network reported in Dyer's work contains more than 4000 interactions between 1218 Y. pestis proteins and 2108 human proteins, which is a much larger dataset than that of Yang's study (Yang et al., 2011a). Only 22 of Yersinia bait proteins are shared and no common interaction exists for these 22 baits in the two datasets, probably and partly due to the fact that different approaches are adopted in the two studies (random $\mathrm{Y} 2 \mathrm{H}$ vs. direct $\mathrm{Y} 2 \mathrm{H})$. In addition to the PPIs revealed by the highthroughput techniques described above, some methods have been developed to predict the interactions between host and microbial pathogens. Over 28,000 interactions between $Y$. pestis and human have been predicted. Some of these interactions can be highly relevant with the pathogenesis of the pathogen and are worthy of further investigation (Krishnadev and Srinivasan, 2011).

\section{PERSPECTIVES}

Comprehensive trans-omic analyses, including genomic, transcriptomic, proteomic, and phenomic data, on E. coli have shown a typical example of a holistic understanding of its cellular physiology and metabolism (Yoon et al., 2012). However, the study is still quantitative and descriptive. Obtaining quantitative and dynamic data, as well as verifying in a large-scale the functions of the differences, is still a long-term task remaining to be tackled.

Most omics reports to date are mainly in vitro studies, and performing in vivo omics studies (such as on pathogen-host interactome in real-time monitoring) is still a challenge in terms of technical development. DNA sequencing and MS technologies prompt us to perform systems studies on pathogenic bacteria, including $Y$. pestis, and help us refine genome annotation (Payne et al., 2010; Schrimpe-Rutledge et al., 2012). As shown above, we have acquired large-scale data for its genomic, transcriptomic, sRNA, and proteomic information; however, we still cannot systematically integrate all these data because the experimental conditions for obtaining these data are not uniformly designed. These fragmented data provide us numerous clues for further studies, and sometimes we cannot decide which targets to choose when faced with many choices. We need to choose the highly and less virulent strains to humans when

\section{REFERENCES}

Achtman, M., Morelli, G., Zhu, P., Wirth, T., Diehl, I., Kusecek, B., et al. (2004). Microevolution and history of the plague bacillus, Yersinia pestis. Proc. Natl. Acad. Sci. U.S.A. 101, 17837-17842.

Achtman, M., Zurth, K., Morelli, G., Torrea, G., Guiyoule, A., and Carniel, E. (1999). Yersinia pestis, the cause of plague, is a recently emerged clone of Yersinia pseudotuberculosis. Proc. Natl. Acad. Sci. U.S.A. 96, 14043-14048.

Anisimov, R., Brem, D., Heesemann, J., and Rakin, A. (2005). Transcriptional regulation of

performing dynamic comparative omics studies, even when comparing with $Y$.pseudotuberculosis, to facilitate a systematic discovery of virulence-associated factors, regulation differences, and host response variations. These data are extremely critical for designing pharmaceuticals, developing diagnostics, and making plague countermeasures.

Schrimpe-Rutledge et al. (2012) have shown a good example of omics-driven genome annotation refinement using transcriptomic and proteomic analyses by comparing the epidemic strain Y. pestis CO92, non-epidemic Y. pestis strain Pestoides F, and Y. pseudotuberculosis PB1. Recently, Ansong et al. gave us another good example of comparative omics study by using genomics, transcriptomics, proteomics and metabolomics to elucidate Yersinia virulence mechanism (Ansong et al., 2012). They not only predicted a network of putative virulence factors consisting of 151 cluster members, including 34 pCD1-encoded proteins, 53 pMT1-encoded ones and 64 chromosomally encoded ones; but also revealed potential virulence roles of 11 pMT1-encoded genes (YPMT1.38c, YPMT1.39c, YPMT1.40c, YPMT1.41c, YPMT1.46Ac, YPMT1.49Ac, YPMT1.49c, YPMT1.52c, YPMT1.55c, YPMT1.88, and YPMT1.89) for larger animals, including humans.

To understand $Y$. pestis virulence using omics in future studies, careful design of cell or animal models and methods of correlating the results to human clinical data are needed. The association of a microbiome and its dynamics in gut or bronchia with plague development is also a considerable challenge to understand the plague from a different angle. To integrate all available omics data from fragmented studies on $Y$. pestis, we need to reannotate the $Y$. pestis genome meticulously, reconstruct its metabolic pathways (Navid and Almaas, 2009), and then integrate all available information for a systems understanding of its life, strengthening the growing importance of bioinformatics and bioinformaticists in the growing datasets provided by Omics research.

\section{ACKNOWLEDGMENTS}

The research on Yersinia pestis in our lab was supported by National Basic Research Program of China (973 Program, 2009CB522600, and 2012CB518700), and the National Natural Science Foundation of China (30930001). This MS was polished by Enpaper, a language editing company.

for $\mathrm{B}$ and $\mathrm{T}$ cell zones of lymph nodes that is independent of the type III secretion system. PLoS Pathog. 2:e86. doi: 10.1371/ journal.ppat.0020086

Bearden, S. W., and Brubaker, R. R. (2010). Recent findings regarding maintenance of enzootic variants of Yersinia pestis in sylvatic reservoirs and their significance in the evolution of epidemic plague. Vector Borne Zoonotic Dis. 10, 85-92.

Bearden, S. W., and Perry, R. D. (1999). The Yfe system of Yersinia pestis transports iron and manganese and is required for full virulence of plague. Mol. Microbiol. 32, 403-414.
Bearden, S. W., Sexton, C., Pare, J., Fowler, J. M., Arvidson, C. G., Yerman, L., et al. (2009). Attenuated enzootic (pestoides) isolates of Yersinia pestis express active aspartase. Microbiology 155, 198-209.

Bearden, S. W., Staggs, T. M., and Perry, R. D. (1998). An ABC transporter system of Yersinia pestis allows utilization of chelated iron by Escherichia coli SAB11. J. Bacteriol. 180, 1135-1147.

Bellows, L. E., Koestler, B. J., Karaba, S. M., Waters, C. M., and Lathem, W. W. (2012). Hfqdependent, co-ordinate control of 
cyclic diguanylate synthesis and catabolism in the plague pathogen Yersinia pestis. Mol. Microbiol. 86, 661-674.

Bergsbaken, T., and Cookson, B. T. (2009). Innate immune response during Yersinia infection: critical modulation of cell death mechanisms through phagocyte activation. J. Leukoc. Biol. 86, 1153-1158.

Bhattacharjee, K., Banerjee, S., and Joshi, S. R. (2012). Diversity of Streptomyces spp. in Eastern Himalayan region - computational RNomics approach to phylogeny. Bioinformation 8, 548-554.

Bozue, J., Mou, S., Moody, K. L., Cote, C. K., Trevino, S., Fritz, D., et al. (2011). The role of the phoPQ operon in the pathogenesis of the fully virulent CO92 strain of Yersinia pestis and the IP32953 strain of Yersinia pseudotuberculosis. Microb. Pathog. 50, 314-321.

Brubaker, R. R. (1991). Factors promoting acute and chronic diseases caused by yersiniae. Clin. Microbiol. Rev. 4, 309-324.

Brubaker, R. R. (2007). Intermediary metabolism, $\mathrm{Na}+$, the low calciumresponse, and acute disease. Adv. Exp. Med. Biol. 603, 116-129.

Calderwood, M. A., Venkatesan, K., Xing, L., Chase, M. R., Vazquez, A., Holthaus, A. M., et al. (2007). Epstein-Barr virus and virus human protein interaction maps. Proc. Natl. Acad. Sci. U.S.A. 104, 7606-7611.

Carniel, E. (1999). The Yersinia highpathogenicity island. Int. Microbiol. 2, 161-167.

Carniel, E. (2001). The Yersinia highpathogenicity island: an iron-uptake island. Microbes Infect. 3, 561-569.

Cathelyn, J. S., Crosby, S. D., Lathem, W. W., Goldman, W. E., and Miller, V. L. (2006). RovA, a global regulator of Yersinia pestis, specifically required for bubonic plague. Proc. Natl. Acad. Sci. U.S.A. 103, 13514-13519.

Cathelyn, J. S., Ellison, D. W., Hinchliffe, S. J., Wren, B. W., and Miller, V. L. (2007). The RovA regulons of Yersinia enterocolitica and Yersinia pestis are distinct: evidence that many RovA-regulated genes were acquired more recently than the core genome. Mol. Microbiol. $66,189-205$.

Cerdeno-Tarraga, A., Thomson, N., and Parkhill, J. (2004). Pathogens in decay. Nat. Rev. Microbiol. 2, 774-775.

Chain, P. S., Carniel, E., Larimer, F. W., Lamerdin, J., Stoutland, P. O., Regala, W. M., et al. (2004). Insights into the evolution of Yersinia pestis through whole-genome comparison with Yersinia pseudotuberculosis. Proc. Natl. Acad. Sci. U.S.A. 101, 13826-13831.

Chapman, E., Farr, G. W., Usaite, R., Furtak, K., Fenton, W. A., Chaudhuri, T. K., et al. (2006). Global aggregation of newly translated proteins in an Escherichia coli strain deficient of the chaperonin GroEL. Proc. Natl. Acad. Sci. U.S.A. 103, 15800-15805.

Charnetzky, W. T., and Shuford, W. W. (1985). Survival and growth of Yersinia pestis within macrophages and an effect of the loss of the 47-megadalton plasmid on growth in macrophages. Infect. Immun. 47, 234-241.

Charusanti, P., Chauhan, S., McAteer, K., Lerman, J. A., Hyduke, D. R., Motin, V. L., et al. (2011). An experimentally-supported genomescale metabolic network reconstruction for Yersinia pestis CO92. BMC Syst. Biol. 5:163. doi: 10.1186/17520509-5-163

Chauvaux, S., Rosso, M. L., Frangeul, L., Lacroix, C., Labarre, L., Schiavo, A., et al. (2007). Transcriptome analysis of Yersinia pestis in human plasma: an approach for discovering bacterial genes involved in septicaemic plague. Microbiology 153, 3112-3124.

Chen, P. E., Cook, C., Stewart, A. C., Nagarajan, N., Sommer, D. D., Pop, M., et al. (2010). Genomic characterization of the Yersinia genus. Genome Biol. 11, R1.

Chen, Z., Li, B., Zhang, J., Qin, L., Zhou, D., Han, Y., et al. (2006). Quorum sensing affects virulenceassociated proteins $\mathrm{F} 1, \mathrm{LcrV}$, KatY and $\mathrm{pH} 6$ etc. of Yersinia pestis as revealed by protein microarraybased antibody profiling. Microbes Infect. 8, 2501-2508.

Chromy, B. A., Choi, M. W., Murphy, G. A., Gonzales, A. D., Corzett, C. H., Chang, B. C., et al. (2005) Proteomic characterization of Yersinia pestis virulence. J. Bacteriol. 187, 8172-8180.

Chromy, B. A., Perkins, J., Heidbrink, J. L., Gonzales, A. D., Murphy, G. A., Fitch, J. P., et al. (2004). Proteomic characterization of host response to Yersinia pestis and near neighbors. Biochem. Biophys. Res. Commun. $320,474-479$.

Comer, J. E., Sturdevant, D. E., Carmody, A. B., Virtaneva, K., Gardner, D., Long, D., et al. (2010). Transcriptomic and innate immune responses to Yersinia pestis in the lymph node during bubonic plague. Infect. Immun. 78, 5086-5098.

Cornelis, G. R. (2002). Yersinia type III secretion: send in the effectors. J. Cell Biol. 158, 401-408.

Cornelis, G. R., Boland, A., Boyd, A. P., Geuijen, C., Iriarte, M., Neyt, C., et al. (1998). The virulence plasmid of Yersinia, an antihost genome. Microbiol. Mol. Biol. Rev. 62, 1315-1352.

Das, R., Dhokalia, A., Huang, X. Z., Hammamieh, R., Chakraborty, N., Lindler, L. E., et al. (2007). Study of proinflammatory responses induced by Yersinia pestis in human monocytes using cDNA arrays. Genes Immun. 8, 308-319.

De Chassey, B., Navratil, V., Tafforeau, L., Hiet, M. S., Aublin-Gex, A., Agaugue, S., et al. (2008). Hepatitis $\mathrm{C}$ virus infection protein network. Mol. Syst. Biol. 4, 230.

De Godoy, L. M., Olsen, J. V., De Souza, G. A., Li, G., Mortensen, P., and Mann, M. (2006). Status of complete proteome analysis by mass spectrometry: SILAC labeled yeast as a model system. Genome Biol. 7, R50.

Deng, W., Burland, V., Plunkett, G. 3rd., Boutin, A., Mayhew, G. F., Liss, P., et al. (2002). Genome sequence of Yersinia pestis KIM. J. Bacteriol. 184, 4601-4611.

Derbise, A., Chenal-Francisque, V., Huon, C., Fayolle, C., Demeure, C. E., Chane-Woon-Ming, B., et al. (2010). Delineation and analysis of chromosomal regions specifying Yersinia pestis. Infect. Immun. 78 3930-3941.

Derbise, A., Chenal-Francisque, V., Pouillot, F., Fayolle, C., Prevost, M. C., Medigue, C., et al. (2007). A horizontally acquired filamentous phage contributes to the pathogenicity of the plague bacillus. Mol. Microbiol. 63, 1145-1157.

Dong, X. Q., Lindler, L. E., and Chu, M. C. (2000). Complete DNA sequence and analysis of an emerging cryptic plasmid isolated from Yersinia pestis. Plasmid 43, 144-148.

Du, Y., Rosqvist, R., and Forsberg, A. (2002). Role of fraction 1 antigen of Yersinia pestis in inhibition of phagocytosis. Infect. Immun. 70, 1453-1460.

Dube, P. H., Handley, S. A., Revell, P. A., and Miller, V. L. (2003). The rovA mutant of Yersinia enterocolitica displays differential degrees of virulence depending on the route of infection. Infect. Immun. 71, 3512-3520.

Dyer, M. D., Murali, T. M., and Sobral, B. W. (2008). The landscape of human proteins interacting with viruses and other pathogens. PLoS Pathog. 4:e32. doi: 10.1371/ journal.ppat.0040032
Dyer, M. D., Neff, C., Dufford, M., Rivera, C. G., Shattuck, D., Bassaganya-Riera, J., et al. (2010). The human-bacterial pathogen protein interaction networks of Bacillus anthracis, Francisella tularensis, and Yersinia pestis. PLoS ONE 5:e12089. doi: 10.1371/journal.pone.0012089

Eisen, M. B., Spellman, P. T., Brown, P. O., and Botstein, D. (1998). Cluster analysis and display of genomewide expression patterns. Proc. Natl. Acad. Sci. U.S.A. 95, 14863-14868.

Ellison, D. W., Lawrenz, M. B., and Miller, V. L. (2004). Invasin and beyond: regulation of Yersinia virulence by RovA. Trends Microbiol. 12, 296-300.

Ellison, D. W., and Miller, V. L. (2006). Regulation of virulence by members of the MarR/SlyA family. Curr. Opin. Microbiol. 9, 153-159.

El Tahir, Y., and Skurnik, M. (2001). YadA, the multifaceted Yersinia adhesin. Int. J. Med. Microbiol. 291, 209-218.

Escolar, L., Perez-Martin, J., and De Lorenzo, V. (1999). Opening the iron box: transcriptional metalloregulation by the Fur protein. J. Bacteriol. 181, 6223-6229.

Fetherston, J. D., Bearden, S. W., and Perry, R. D. (1996). YbtA, an AraC-type regulator of the Yersinia pestis pesticin/yersiniabactin receptor. Mol. Microbiol. 22, 315-325.

Fetherston, J. D., and Perry, R. D. (1994). The pigmentation locus of Yersinia pestis KIM6+ is flanked by an insertion sequence and includes the structural genes for pesticin sensitivity and HMWP2. Mol. Microbiol. 13, 697-708.

Fukuto, H. S., Svetlanov, A., Palmer L. E., Karzai, A. W., and Bliska, J. B. (2010). Global gene expression profiling of Yersinia pestis replicating inside macrophages reveals the roles of a putative stress-induced operon in regulating type III secretion and intracellular cell division. Infect. Immun. 78, 3700-3715.

Galindo, C. L., Moen, S. T., Kozlova, E. V., Sha, J., Garner, H. R., Agar, S. L., et al. (2009). Comparative analyses of transcriptional profiles in mouse organs using a pneumonic plague model after infection with wild-type Yersinia pestis $\mathrm{CO} 92$ and Its braun lipoprotein mutant. Comp. Funct. Genomics 2009, 914762.

Gao, H., Zhou, D., Li, Y., Guo, Z., Han, Y., Song, Y., et al. (2008). The ironresponsive Fur regulon in Yersinia pestis. J. Bacteriol. 190, 3063-3075.

Geng, J., Song, Y., Yang, L., Feng, Y., Qiu, Y., Li, G., et al. (2009). Involvement of the posttranscriptional regulator $\mathrm{Hfq}$ 
in Yersinia pestis virulence. PLoS ONE 4:e6213. doi: 10.1371/ journal.pone.0006213

Gogarten, J. P., Doolittle, W. F., and Lawrence, J. G. (2002). Prokaryotic evolution in light of gene transfer. Mol. Biol. Evol. 19, 2226-2238.

Gonzalez, M. D., Lichtensteiger, C. A., Caughlan, R., and Vimr, E. R. (2002). Conserved filamentous prophage in Escherichia coli O18:K1:H7 and Yersinia pestis biovar orientalis. J. Bacteriol. 184, 6050-6055.

Goodman, A. L., and Lory, S. (2004). Analysis of regulatory networks in Pseudomonas aeruginosa by genomewide transcriptional profiling. Curr. Opin. Microbiol. 7, 39-44.

Grabenstein, J. P., Fukuto, H. S., Palmer, L. E., and Bliska, J. B. (2006). Characterization of phagosome trafficking and identification of PhoP-regulated genes important for survival of Yersinia pestis in macrophages. Infect. Immun. 74, 3727-3741.

Groisman, E. A. (2001). The pleiotropic two-component regulatory system PhoP-PhoQ. J. Bacteriol. 183, 1835-1842.

Guell, M., Yus, E., Lluch-Senar, M., and Serrano, L. (2011). Bacterial transcriptomics: what is beyond the RNA horiz-ome? Nat. Rev. Microbiol. 9, 658-669.

Guinet, F., Ave, P., Jones, L., Huerre, M., and Carniel, E. (2008). Defective innate cell response and lymph node infiltration specify Yersinia pestis infection. PLOS ONE 3:e1688. doi: 10.1371/journal.pone.0001688

Han, Y., Qiu, J., Guo, Z., Gao, H., Song, Y., Zhou, D., et al. (2007). Comparative transcriptomics in Yersinia pestis: a global view of environmental modulation of gene expression. BMC Microbiol. 7:96. doi: 10.1186/1471-2180-7-96

Han, Y., Zhou, D., Pang, X., Song, Y., Zhang, L., Bao, J., et al. (2004). Microarray analysis of temperatureinduced transcriptome of Yersinia pestis. Microbiol. Immunol. 48, 791-805.

Han, Y., Zhou, D., Pang, X., Zhang, L., Song, Y., Tong, Z., et al. (2005a). Comparative transcriptome analysis of Yersinia pestis in response to hyperosmotic and highsalinity stress. Res. Microbiol. 156, 403-415.

Han, Y., Zhou, D., Pang, X., Zhang, L., Song, Y., Tong, Z., et al. (2005b). DNA microarray analysis of the heat- and cold-shock stimulons in Yersinia pestis. Microbes Infect. 7, 335-348.
Hartler, J., Tharakan, R., Kofeler, H. C., Graham, D. R., and Thallinger, G. G. (2012). Bioinformatics tools and challenges in structural analysis of lipidomics MS/MS data. Brief Bioinform. doi: 10.1093/bib/bbs030. [Epub ahead of print]

Heroven, A. K., Nagel, G., Tran, H. J., Parr, S., and Dersch, P. (2004). RovA is autoregulated and antagonizes $\mathrm{H}$ NS-mediated silencing of invasin and rovA expression in Yersinia pseudotuberculosis. Mol. Microbiol. 53, 871-888.

Herrero, J., and Dopazo, J. (2002). Combining hierarchical clustering and self-organizing maps for exploratory analysis of gene expression patterns. J. Proteome Res. 1, 467-470.

Heurlier, K., Denervaud, V., and Haas, D. (2006). Impact of quorum sensing on fitness of Pseudomonas aeruginosa. Int. J. Med. Microbiol. 296, 93-102.

Hinnebusch, B. J., Rudolph, A. E., Cherepanov, P., Dixon, J. E., Schwan, T. G., and Forsberg, A. (2002). Role of Yersinia murine toxin in survival of Yersinia pestis in the midgut of the flea vector. Science 296, 733-735.

Hinnebusch, J., Cherepanov, P., Du, Y., Rudolph, A., Dixon, J. D., Schwan, T., et al. (2000). Murine toxin of Yersinia pestis shows phospholipase $\mathrm{D}$ activity but is not required for virulence in mice. Int. J. Med. Microbiol. 290, 483-487.

Hitchen, P. G., Prior, J. L., Oyston, P. C., Panico, M., Wren, B. W., Titball, R. W., et al. (2002). Structural characterization of lipo-oligosaccharide (LOS) from Yersinia pestis: regulation of LOS structure by the PhoPQ system. Mol. Microbiol. 44, 1637-1650.

Hixson, K. K., Adkins, J. N., Baker, S. E., Moore, R. J., Chromy, B. A., Smith, R. D., et al. (2006). Biomarker candidate identification in Yersinia pestis using organismwide semiquantitative proteomics. J. Proteome Res. 5, 3008-3017.

Hoffmann, R., Van Erp, K., Trulzsch, K., and Heesemann, J. (2004). Transcriptional responses of murine macrophages to infection with Yersinia enterocolitica. Cell. Microbiol. 6, 377-390.

Holmes, C., McDonald, F., Jones, M., Ozdemir, V., and Graham, J. E. (2010). Standardization and omics science: technical and social dimensions are inseparable and demand symmetrical study. OMICS 14, 327-332.

Horler, R. S., and Vanderpool, C. K. (2009). Homologs of the small
RNA SgrS are broadly distributed in enteric bacteria but have diverged in size and sequence. Nucleic Acids Res. $37,5465-5476$

Huang, X. Z., and Lindler, L. E. (2004) The $\mathrm{pH} 6$ antigen is an antiphagocytic factor produced by Yersinia pestis independent of Yersinia outer proteins and capsule antigen. Infect. Immun. 72, 7212-7219.

Jabbour, R. E., Wade, M. M. Deshpande, S. V., Stanford, M. F., Wick, C. H., Zulich, A. W., et al. (2010). Identification of Yersinia pestis and Escherichia coli strains by whole cell and outer membrane protein extracts with mass spectrometry-based proteomics. J. Proteome Res. 9, 3647-3655.

Kawahara, K., Tsukano, H., Watanabe, H., Lindner, B., and Matsuura, M. (2002). Modification of the structure and activity of lipid A in Yersinia pestis lipopolysaccharide by growth temperature. Infect. Immun. 70, 4092-4098.

Kim, T. J., Chauhan, S., Motin, V. L. Goh, E. B., Igo, M. M., and Young, G. M. (2007). Direct transcriptional control of the plasminogen activator gene of Yersinia pestis by the cyclic AMP receptor protein. J. Bacteriol. 189, 8890-8900.

Kindrachuk, J., Arsenault, R., Kusalik, A., Kindrachuk, K. N., Trost, B. Napper, S., et al. (2012). Systems kinomics demonstrates congo basin monkeypox virus infection selectively modulates host cell signaling responses as compared to west african monkeypox virus. Mol. Cell. Proteomics 11:M111.015701. doi: 10.1074/mcp.M111.015701

Knox, S. S. (2010). From 'omics' to complex disease: a systems biology approach to gene-environment interactions in cancer. Cancer Cell Int. 10, 11.

Kolker, E. (2009). OMICS: 2009, 2010 and beyond. OMICS 13, 451.

Koo, J. T., Alleyne, T. M., Schiano, C. A., Jafari, N., and Lathem, W. W (2011). Global discovery of small RNAs in Yersinia pseudotuberculosis identifies Yersinia-specific small, noncoding RNAs required for virulence. Proc. Natl. Acad. Sci. U.S.A. 108, E709-E717.

Krishnadev, O., and Srinivasan, N (2011). Prediction of proteinprotein interactions between human host and a pathogen and its application to three pathogenic bacteria. Int. J. Biol. Macromol. 48 613-619.

Kukkonen, M., Suomalainen, M., Kyllonen, P., Lahteenmaki, K., Lang, H., Virkola, R., et al. (2004). Lack of $\mathrm{O}$-antigen is essential for plasminogen activation by Yersinia pestis and Salmonella enterica. Mol. Microbiol. 51, 215-225.

Lacount, D. J., Vignali, M., Chettier, R. Phansalkar, A., Bell, R., Hesselberth, J. R., et al. (2005). A protein interaction network of the malaria parasite Plasmodium falciparum. Nature 438 103-107.

Lathem, W. W., Crosby, S. D., Miller, V. L., and Goldman, W. E. (2005). Progression of primary pneumonic plague: a mouse model of infection, pathology, and bacterial transcriptional activity. Proc. Natl. Acad. Sci. U.S.A. 102, 17786-17791.

Lathem, W. W., Price, P. A., Miller, V. L., and Goldman, W. E. (2007). A plasminogen-activating protease specifically controls the development of primary pneumonic plague. Science 315, 509-513.

Lawson, J. N., Lyons, C. R., and Johnston, S. A. (2006). Expression profiling of Yersinia pestis during mouse pulmonary infection. DNA Cell Biol. 25, 608-616.

Lee, H. Y., Cho, S. A., Lee, I. S., Park, J. H., Seok, S. H., Baek, M. W., et al. (2007). Evaluation of phoP and rpoS mutants of Salmonella enterica serovar Typhi as attenuated typhoid vaccine candidates: virulence and protective immune responses in intranasally immunized mice. FEMS Immunol. Med. Microbiol. 51, 310-318.

Lemaitre, N., Sebbane, F., Long, D., and Hinnebusch, B. J. (2006). Yersinia pestis YopJ suppresses tumor necrosis factor alpha induction and contributes to apoptosis of immune cells in the lymph node but is not required for virulence in a rat model of bubonic plague. Infect. Immun. 74, 5126-5131.

Levesque, D., Reymond, C. and Perreault, J. P. (2012) Characterization of the trans Watson-Crick GU base pair located in the catalytic core of the antigenomic HDV ribozyme. PLOS ONE 7:e40309. doi: 10.1371/journal.pone.0040309

Li, B., Du, C., Zhou, L., Bi, Y., Wang, X., Wen, L., et al. (2012). Humoral and cellular immune responses to Yersinia pestis infection in longterm recovered plague patients Clin. Vaccine Immunol. 19, 228-234.

Li, B., Tan, Y., Guo, J., Cui, B., Wang, Z., Wang, H., et al. (2011). Use of protein microarray to identify gene expression changes of Yersinia pestis at different temperatures. Can.J. Microbiol. 57, 287-294.

Li, S., Armstrong, C. M., Bertin, N., Ge, H., Milstein, S., Boxem, M., et al. (2004). A map of the interactome 
network of the metazoan C. elegans. Science 303, 540-543.

Li, Y., Dai, E., Cui, Y., Li, M., Zhang, Y., Wu, M., et al. (2008a). Different region analysis for genotyping Yersinia pestis isolates from China. PLoS ONE 3:e2166. doi: 10.1371/journal.pone.0002166

Li, Y. L., Gao, H., Qin, L., Li, B., Han, Y. P., Guo, Z. B., et al. (2008b). Identification and characterization of PhoP regulon members in Yersinia pestis biovar Microtus. BMC Genomics 9:143. doi: 10.1186/1471-2164-9-143

Lin, J. S., Kummer, L. W., Szaba, F. M., and Smiley, S. T. (2011). IL-17 contributes to cell-mediated defense against pulmonary Yersinia pestis infection. J. Immunol. 186, 1675-1684.

Lindler, L. E., Klempner, M. S., and Straley, S. C. (1990). Yersinia pestis pH 6 antigen: genetic, biochemical, and virulence characterization of a protein involved in the pathogenesis of bubonic plague. Infect. Immun. 58, 2569-2577.

Lindler, L. E., Plano, G. V., Burland, V., Mayhew, G. F., and Blattner, F. R. (1998). Complete DNA sequence and detailed analysis of the Yersinia pestis KIM5 plasmid encoding murine toxin and capsular antigen. Infect. Immun. 66, 5731-5742.

Liu, H., Wang, H., Qiu, J., Wang, X., Guo, Z., Qiu, Y., et al. (2009). Transcriptional profiling of a mice plague model: insights into interaction between Yersinia pestis and its host. J. Basic Microbiol. 49, 92-99.

Lukaszewski, R. A., Kenny, D. J., Taylor, R., Rees, D. G., Hartley, M. G., and Oyston, P. C. (2005). Pathogenesis of Yersinia pestis infection in BALB/C mice: effects on host macrophages and neutrophils. Infect. Immun. 73, 7142-7150.

Lv, H., and Henderson, J. P. (2011). Yersinia high pathogenicity island genes modify the Escherichia coli primary metabolome independently of siderophore production. J. Proteome Res. 10, 5547-5554.

Mahapatra, A. (2010). Omics in the postgenomic era. ACS Chem. Biol. 5, 343-344.

Makoveichuk, E., Cherepanov, P., Lundberg, S., Forsberg, A., and Olivecrona, G. (2003). pH6 antigen of Yersinia pestis interacts with plasma lipoproteins and cell membranes. J. Lipid Res. 44, 320-330.

Marioni, J. C., Mason, C. E., Mane, S. M., Stephens, M., and Gilad, Y. (2008). RNA-seq: an assessment of technical reproducibility and comparison with gene expression arrays. Genome Res. 18, 1509-1517.

Marketon, M. M., Depaolo, R. W., Debord, K. L., Jabri, B., and Schneewind, O. (2005). Plague bacteria target immune cells during infection. Science 309, 1739-1741.

Montminy, S. W., Khan, N., McGrath, S., Walkowicz, M. J., Sharp, F., Conlon, J. E., et al. (2006). Virulence factors of Yersinia pestis are overcome by a strong lipopolysaccharide response. Nat. Immunol. 7, 1066-1073.

Moran, N. A. (2002). Microbial minimalism: genome reduction in bacterial pathogens. Cell 108, 583-586.

Morelli, G., Song, Y., Mazzoni, C. J., Eppinger, M., Roumagnac, P., Wagner, D. M., et al. (2010). Yersinia pestis genome sequencing identifies patterns of global phylogenetic diversity. Nat. Genet. 42, 1140-1143.

Morrison, N., Cochrane, G., Faruque, N., Tatusova, T., Tateno, Y., Hancock, D., et al. (2006). Concept of sample in OMICS technology. OMICS 10, 127-137.

Mortazavi, A., Williams, B. A., McCue, K., Schaeffer, L., and Wold, B. (2008). Mapping and quantifying mammalian transcriptomes by RNA-Seq. Nat. Methods 5, 621-628.

Motin, V. L., Georgescu, A. M., Fitch, J. P., Gu, P. P., Nelson, D. O., Mabery, S. L., et al. (2004). Temporal global changes in gene expression during temperature transition in Yersinia pestis. J. Bacteriol. 186, 6298-6305.

Mukherjee, S., Keitany, G., Li, Y., Wang, Y., Ball, H. L., Goldsmith, E. J., et al. (2006). Yersinia YopJ acetylates and inhibits kinase activation by blocking phosphorylation. Science 312, 1211-1214.

Nagel, G., Lahrz, A., and Dersch, P. (2001). Environmental control of invasin expression in Yersinia pseudotuberculosis is mediated by regulation of RovA, a transcriptional activator of the SlyA/Hor family. Mol. Microbiol. 41, 1249-1269.

Nakajima, R., and Brubaker, R. R. (1993). Association between virulence of Yersinia pestis and suppression of gamma interferon and tumor necrosis factor alpha. Infect. Immun. 61, 23-31.

Narberhaus, F., and Vogel, J. (2009). Regulatory RNAs in prokaryotes: here, there and everywhere. Mol. Microbiol. 74, 261-269.

Navid, A. (2011). Applications of system-level models of metabolism for analysis of bacterial physiology and identification of new drug targets. Brief Funct. Genomics 10, 354-364.
Navid, A., and Almaas, E. (2009). Genome-scale reconstruction of the metabolic network in Yersinia pestis, strain 91001. Mol. Biosyst. 5, 368-375.

Ning, M., and Lo, E. H. (2010). Opportunities and challenges in Omics. Transl. Stroke Res. 1, 233-237.

Ochman, H. (2005). Genomes on the shrink. Proc. Natl. Acad. Sci. U.S.A. 102, 11959-11960.

Ogata, Y., Chikayama, E., Morioka, Y., Everroad, R. C., Shino, A., Matsushima, A., et al. (2012). ECOMICS: a web-based toolkit for investigating the biomolecular web in ecosystems using a trans-omics approach. PLoS ONE 7:e30263. doi 10.1371/journal.pone.0030263

O’Loughlin, J. L., Spinner, J. L., Minnich, S. A., and Kobayashi, S. D. (2010). Yersinia pestis twocomponent gene regulatory systems promote survival in human neutrophils. Infect. Immun. 78, 773-782.

Olsen, A. N., Mundy, J., and Skriver, K. (2002). Peptomics, identification of novel cationic Arabidopsis peptides with conserved sequence motifs. In Silico Biol. 2, 441-451.

Olsen, J. V., De Godoy, L. M., Li, G., Macek, B., Mortensen, P., Pesch, R., et al. (2005). Parts per million mass accuracy on an Orbitrap mass spectrometer via lock mass injection into a C-trap. Mol. Cell. Proteomics 4, 2010-2021.

Oyston, P. C., Dorrell, N., Williams, K. Li, S. R., Green, M., Titball, R. W. et al. (2000). The response regulator PhoP is important for survival under conditions of macrophageinduced stress and virulence in Yersinia pestis. Infect. Immun. 68, 3419-3425.

Pallen, M. J., and Wren, B. W. (2007). Bacterial pathogenomics. Nature 449, 835-842

Parkhill, J., Wren, B. W., Thomson, N. R., Titball, R. W., Holden, M. T., Prentice, M. B., et al. (2001). Genome sequence of Yersinia pestis, the causative agent of plague. Nature $413,523-527$.

Parrish, J. R., Yu, J., Liu, G., Hines, J. A. Chan, J. E., Mangiola, B. A., et al. (2007). A proteome-wide protein interaction map for Campylobacter jejuni. Genome Biol. 8, R130

Payne, D., Tatham, D., Williamson, E. D., and Titball, R. W. (1998). The $\mathrm{pH} 6$ antigen of Yersinia pestis binds to betal-linked galactosyl residues in glycosphingolipids. Infect. Immun. 66, 4545-4548.

Payne, S. H., Huang, S. T., and Pieper, R. (2010). A proteogenomic update to Yersinia: enhancing genome annotation. BMC Genomics 11:460. doi: 10.1186/1471-2164-11-460

Pendrak, M. L., and Perry, R. D. (1991). Characterization of a hemin-storage locus of Yersinia pestis. Biol. Met. 4, 41-47.

Peng, J., Elias, J. E., Thoreen, C. C., Licklider, L. J., and Gygi, S. P. (2003). Evaluation of multidimensional chromatography coupled with tandem mass spectrometry (LC/LC-MS/MS) for large-scale protein analysis: the yeast proteome. J. Proteome Res. 2, 43-50.

Perez, J. C., and Groisman, E. A. (2009). Transcription factor function and promoter architecture govern the evolution of bacterial regulons. Proc. Natl. Acad. Sci. U.S.A. 106, 4319-4324.

Perez, J. C., Shin, D., Zwir, I., Latifi, T., Hadley, T. J., and Groisman, E. A. (2009). Evolution of a bacterial regulon controlling virulence and $\mathrm{Mg}(2+)$ homeostasis. PLoS Genet. 5:e1000428. doi: 10.1371/journal.pgen.1000428

Perez-Gutierrez, C., Llobet, E., Llompart, C. M., Reines, M. and Bengoechea, J. A. (2010). Role of lipid A acylation in Yersinia enterocolitica virulence. Infect. Immun. 78, 2768-2781.

Perry, R. D., and Fetherston, J. D. (1997). Yersinia pestis-etiologic agent of plague. Clin. Microbiol. Rev. 10, 35-66.

Perry, R. D., Straley, S. C., Fetherston, J. D., Rose, D. J., Gregor, J., and Blattner, F. R. (1998). DNA sequencing and analysis of the low-Ca2+response plasmid pCD1 of Yersinia pestis KIM5. Infect. Immun. 66, 4611-4623.

Pieper, R., Huang, S. T., Clark, D. J., Robinson, J. M., Parmar, P. P., Alami, H., et al. (2008). Characterizing the dynamic nature of the Yersinia pestis periplasmic proteome in response to nutrient exhaustion and temperature change. Proteomics 8, 1442-1458.

Pieper, R., Huang, S. T., Parmar, P. P., Clark, D. J., Alami, H., Fleischmann, R. D., et al. (2010). Proteomic analysis of iron acquisition, metabolic and regulatory responses of Yersinia pestis to iron starvation. BMC Microbiol. 10:30. doi: 10.1186/1471-2180-10-30

Pieper, R., Huang, S. T., Robinson, J. M., Clark, D. J., Alami, H., Parmar, P. P., et al. (2009). Temperature and growth phase influence the outer-membrane proteome and the expression of a type VI secretion system in Yersinia pestis. Microbiology 155, 498-512. 
Ponnusamy, D., Hartson, S. D., and Clinkenbeard, K. D. (2011). Intracellular Yersinia pestis expresses general stress response and tellurite resistance proteins in mouse macrophages. Vet. Microbiol. 150, 146-151.

Pouillot, F., Fayolle, C., and Carniel, E. (2008). Characterization of chromosomal regions conserved in Yersinia pseudotuberculosis and lost by Yersinia pestis. Infect. Immun. 76, 4592-4599.

Price, P. A., Jin, J., and Goldman, W. E. (2012). Pulmonary infection by Yersinia pestis rapidly establishes a permissive environment for microbial proliferation. Proc. Natl. Acad. Sci. U.S.A. 109, 3083-3088.

Pujol, C., and Bliska, J. B. (2003). The ability to replicate in macrophages is conserved between Yersinia pestis and Yersinia pseudotuberculosis. Infect. Immun. 71, 5892-5899.

Pujol, C., Klein, K. A., Romanov, G. A., Palmer, L. E., Cirota, C., Zhao, Z., et al. (2009). Yersinia pestis can reside in autophagosomes and avoid xenophagy in murine macrophages by preventing vacuole acidification. Infect. Immun. 77, 2251-2261.

Qiu, J., Zhou, D., Han, Y., Zhang, L., Tong, Z., Song, Y., et al. (2005). Global gene expression profile of Yersinia pestis induced by streptomycin. FEMS Microbiol. Lett. 243, 489-496.

Qiu, J., Zhou, D., Qin, L., Han, Y., Wang, X., Du, Z., et al. (2006). Microarray expression profiling of Yersinia pestis in response to chloramphenicol. FEMS Microbiol. Lett. 263, 26-31.

Qu, Y., Bi, L., Ji, X., Deng, Z., Zhang, H., Yan, Y., et al. (2012). Identification by cDNA cloning of abundant sRNAs in a human-avirulent Yersinia pestis strain grown under five different growth conditions. Future Microbiol. 7, 535-547.

Rempe, K. A., Hinz, A. K., and Vadyvaloo, V. (2012). Hfq regulates biofilm gut blockage that facilitates flea-borne transmission of Yersinia pestis. J. Bacteriol. 194, 2036-2040.

Revell, P. A., and Miller, V. L. (2000). A chromosomally encoded regulator is required for expression of the Yersinia enterocolitica inv gene and for virulence. Mol. Microbiol. 35, 677-685.

Riding, G. A., Jones, A., Holland, M. K., Hill, J. R., and Lehnert, S. A. (2008). Proteomic analysis of bovine conceptus fluids during early pregnancy. Proteomics 8, 160-177.

Rogers, J. V., Choi, Y. W., Giannunzio, L. F., Sabourin, P. J., Bornman, D. M., Blosser, E. G., et al. (2007).
Transcriptional responses in spleens from mice exposed to Yersinia pestis CO92. Microb. Pathog. 43, 67-77.

Rosqvist, R., Skurnik, M., and WolfWatz, H. (1988). Increased virulence of Yersinia pseudotuberculosis by two independent mutations. Nature 334, 522-524.

Rual, J. F., Venkatesan, K., Hao, T., Hirozane-Kishikawa, T., Dricot, A., Li, N., et al. (2005). Towards a proteome-scale map of the human protein-protein interaction network. Nature 437, 1173-1178.

Schrimpe-Rutledge, A. C., Jones, M. B., Chauhan, S., Purvine, S. O., Sanford, J. A., Monroe, M. E., et al. (2012). Comparative omics-driven genome annotation refinement: application across Yersiniae. PLoS ONE 7:e33903. doi: 10.1371/journal.pone.0033903

Sebbane, F., Devalckenaere, A., Foulon, J., Carniel, E., and Simonet, M. (2001). Silencing and reactivation of urease in Yersinia pestis is determined by one $\mathrm{G}$ residue at a specific position in the ureD gene. Infect. Immun. 69, 170-176.

Sebbane, F., Gardner, D., Long, D., Gowen, B. B., and Hinnebusch, B. J. (2005). Kinetics of disease progression and host response in a rat model of bubonic plague. Am. J. Pathol. 166, 1427-1439.

Sebbane, F., Jarrett, C., Gardner, D., Long, D., and Hinnebusch, B. J. (2010). Role of the Yersinia pestis yersiniabactin iron acquisition system in the incidence of flea-borne plague. PLoS ONE 5:e14379. doi: 10.1371/journal.pone.0014379

Sebbane, F., Jarrett, C. O., Gardner, D., Long, D., and Hinnebusch, B. J. (2006a). Role of the Yersinia pestis plasminogen activator in the incidence of distinct septicemic and bubonic forms of flea-borne plague. Proc. Natl. Acad. Sci. U.S.A. 103, 5526-5530.

Sebbane, F., Lemaitre, N., Sturdevant, D. E., Rebeil, R., Virtaneva, K., Porcella, S. F., et al. (2006b). Adaptive response of Yersinia pestis to extracellular effectors of innate immunity during bubonic plague. Proc. Natl. Acad. Sci. U.S.A. 103, 11766-11771.

Simonet, M., Riot, B., Fortineau, N., and Berche, P. (1996). Invasin production by Yersinia pestis is abolished by insertion of an IS200-like element within the inv gene. Infect. Immun. 64, 375-379.

Simpson, W. J., Thomas, R. E., and Schwan, T. G. (1990). Recombinant capsular antigen (fraction 1) from Yersinia pestis induces a protective antibody response in BALB/c mice. Am. J. Trop. Med. Hyg. 43, 389-396.

Sodeinde, O. A., and Goguen, J. D. (1988). Genetic analysis of the 9.5-kilobase virulence plasmid of Yersinia pestis. Infect. Immun. 56, 2743-2748.

Sodeinde, O. A., Subrahmanyam, Y. V., Stark, K., Quan, T., Bao, Y., and Goguen, J. D. (1992). A surface protease and the invasive character of plague. Science 258, 1004-1007.

Song, Y., Tong, Z., Wang, J., Wang, L., Guo, Z., Han, Y., et al. (2004). Complete genome sequence of Yersinia pestis strain 91001, an isolate avirulent to humans. DNA Res. 11, 179-197.

Stelzl, U., Worm, U., Lalowski, M., Haenig, C., Brembeck, F. H., Goehler, H., et al. (2005). A human protein-protein interaction network: a resource for annotating the proteome. Cell 122, 957-968.

Straley, S. C., and Harmon, P. A (1984). Growth in mouse peritoneal macrophages of Yersinia pestis lacking established virulence determinants. Infect. Immun. 45, 649-654.

Subrahmanyam, Y. V., Yamaga, S., Prashar, Y., Lee, H. H., Hoe, N. P., Kluger, Y., et al. (2001). RNA expression patterns change dramatically in human neutrophils exposed to bacteria. Blood 97, 2457-2468.

Sun, W., Six, D., Kuang, X., Roland, K. L., Raetz, C. R., and Curtiss, R. 3rd. (2011). A live attenuated strain of Yersinia pestis KIM as a vaccine against plague. Vaccine 29, 2986-2998.

Sun, Y. C., Hinnebusch, B. J., and Darby, C. (2008). Experimental evidence for negative selection in the evolution of a Yersinia pestis pseudogene. Proc. Natl. Acad. Sci. U.S.A. 105, 8097-8101.

Superti, F., Pietrantoni, A., Di Biase, A. M., Longhi, C., Valenti, P., and Tinari, A. (2005). Inv-mediated apoptosis of epithelial cells infected with enteropathogenic Yersinia: a protective effect of lactoferrin. Res. Microbiol. 156, 728-737.

Telepnev, M. V., Klimpel, G. R., Haithcoat, J., Knirel, Y. A., Anisimov, A. P., and Motin, V. L. (2009). Tetraacylated lipopolysaccharide of Yersinia pestis can inhibit multiple Toll-like receptormediated signaling pathways in human dendritic cells. J. Infect. Dis. 200, 1694-1702.

Thompson, A., Rowley, G., Alston, M., Danino, V., and Hinton, J. C. (2006). Salmonella transcriptomics: relating regulons, stimulons and regulatory networks to the process of infection. Curr. Opin. Microbiol. 9, 109-116.
Tong, Z., Zhou, D., Song, Y., Zhang, L., Pei, D., Han, Y., et al. (2005). Pseudogene accumulation might promote the adaptive microevolution of Yersinia pestis. J. Med. Microbiol. 54, 259-268.

Tuohy, K. M., Gougoulias, C., Shen, Q., Walton, G., Fava, F., and Ramnani, P. (2009). Studying the human gut microbiota in the trans-omics era-focus on metagenomics and metabonomics. Curr. Pharm. Des. 15, 1415-1427.

Turnbull, J. E., and Sasisekharan, R. (2010). Glycomics: technologies taming a frontier omics field. OMICS 14, 385-387.

Uetz, P., Dong, Y. A., Zeretzke, C., Atzler, C., Baiker, A., Berger, B., et al. (2006). Herpesviral protein networks and their interaction with the human proteome. Science 311, 239-242.

Vadyvaloo, V., Jarrett, C., Sturdevant, D. E., Sebbane, F., and Hinnebusch, B. J. (2010). Transit through the flea vector induces a pretransmission innate immunity resistance phenotype in Yersinia pestis. PLoS Pathog. 6:e1000783. doi: 10.1371/journal.ppat.1000783

Van Erp, K., Dach, K., Koch, I., Heesemann, J., and Hoffmann, R (2006). Role of strain differences on host resistance and the transcriptional response of macrophages to infection with Yersinia enterocolitica. Physiol. Genomics 25, 75-84.

Viboud, G. I., and Bliska, J. B. (2005). Yersinia outer proteins: role in modulation of host cell signaling responses and pathogenesis. Annu. Rev. Microbiol. 59, 69-89.

Viola, R. E., Yerman, L., Fowler, J. M., Arvidson, C. G., and Brubaker, R. R. (2008). A missense mutation causes aspartase deficiency in Yersinia pestis. Microbiology 154, 1271-1280.

Vogel, J., and Luisi, B. F. (2011). Hfq and its constellation of RNA. Nat. Rev. Microbiol. 9, 578-589.

Wadler, C. S., and Vanderpool, C. K. (2007). A dual function for a bacterial small RNA: SgrS performs base pairing-dependent regulation and encodes a functional polypeptide. Proc. Natl. Acad. Sci. U.S.A. 104, 20454-20459.

Wadler, C. S., and Vanderpool, C. K. (2009). Characterization of homologs of the small RNA SgrS reveals diversity in function. Nucleic Acids Res. 37, 5477-5485.

Wall, D. B., Kachman, M. T., Gong, S., Hinderer, R., Parus, S., Misek, D. E., et al. (2000). Isoelectric focusing nonporous RP HPLC: 
a two-dimensional liquid-phase separation method for mapping of cellular proteins with identification using MALDI-TOF mass spectrometry. Anal. Chem. 72, 1099-1111.

Wang, Y., Liang, Q., Hu, P., and Luo, G. (2008). [Application of chromatography and related techniques in quality evaluation of traditional Chinese medicine]. Se Pu 26, 136-141.

Wang, Z., Gerstein, M., and Snyder, M. (2009). RNA-Seq: a revolutionary tool for transcriptomics. Nat. Rev. Genet. 10, 57-63.

Washburn, M. P., Wolters, D., and Yates, J. R. 3rd. (2001). Large-scale analysis of the yeast proteome by multidimensional protein identification technology. Nat. Biotechnol. 19, 242-247.

Waters, L. S., and Storz, G. (2009). Regulatory RNAs in bacteria. Cell 136, 615-628.

Welch, T. J., Fricke, W. F., McDermott, P. F., White, D. G., Rosso, M. L., Rasko, D. A., et al. (2007). Multiple antimicrobial resistance in plague: an emerging public health risk. PLOS ONE 2:e309. doi: 10.1371/journal.pone.0000309

Wild, C. P. (2010). OMICS technologies: an opportunity for "two-way" translation from basic science to both clinical and population-based research. Occup. Environ. Med. 67, 75-76.

Wilhelm, B. T., and Landry, J. R. (2009). RNA-Seq-quantitative measurement of expression through massively parallel RNA-sequencing. Methods 48, 249-257.

Yang, F., Ke, Y., Tan, Y., Bi, Y., Shi, Q., Yang, H., et al. (2010). Cell membrane is impaired, accompanied by enhanced type III secretion system expression in Yersinia pestis deficient in RovA regulator. PLoS ONE 5:e12840. doi: 10.1371/journal.pone.0012840
Yang, H., Ke, Y., Wang, J., Tan, Y., Myeni, S. K., Li, D., et al. (2011a). Insight into bacterial virulence mechanisms against host immune response via the Yersinia pestishuman protein-protein interaction network. Infect. Immun. 79, 4413-4424.

Yang, X., Jiao, R., Yang, L., Wu, L. P., Li, Y. R., and Wang, J. (2011b). [New-generation high-throughput technologies based 'omics' research strategy in human disease]. $Y i$ Chuan 33, 829-846.

Yoon, S. H., Han, M. J., Jeong, H., Lee, C. H., Xia, X. X., Lee, D. H., et al. (2012). Comparative multi-omics systems analysis of Escherichia coli strains B and K-12. Genome Biol. 13, R37.

Zav'yalov, V. P., Abramov, V. M., Cherepanov, P. G., Spirina, G. V., Chernovskaya, T. V., Vasiliev, A. M., et al. (1996). pH6 antigen (PsaA protein) of Yersinia pestis, a novel bacterial Fc-receptor. FEMS Immunol. Med. Microbiol. 14, 53-57.

Zhan, L., Han, Y., Yang, L., Geng, J., Li, Y., Gao, H., et al. (2008). The cyclic AMP receptor protein, CRP, is required for both virulence and expression of the minimal CRP regulon in Yersinia pestis biovar microtus. Infect. Immun. 76, 5028-5037.

Zhan, L., Yang, L., Zhou, L., Li, Y., Gao, H., Guo, Z., et al. (2009). Direct and negative regulation of the sycOypkA-ypoJ operon by cyclic AMP receptor protein (CRP) in Yersinia pestis. BMC Microbiol. 9:178. doi: 10.1186/1471-2180-9-178

Zhang, A., Wassarman, K. M., Rosenow, C., Tjaden, B. C., Storz, G., and Gottesman, S. (2003). Global analysis of small RNA and mRNA targets of Hfq. Mol. Microbiol. 50, 1111-1124.

Zhang, C. G., Chromy, B. A., and McCutchen-Maloney, S. L. (2005a). Host-pathogen interactions: a proteomic view. Expert Rev. Proteomics 2, 187-202.

Zhang, C. G., Gonzales, A. D., Choi, M. W., Chromy, B. A., Fitch, J. P., and McCutchen-Maloney, S. L. (2005b) Subcellular proteomic analysis of host-pathogen interactions using human monocytes exposed to Yersinia pestis and Yersinia pseudotuberculosis. Proteomics 5, 1877-1888.

Zheng, D., Constantinidou, C., Hobman, J. L., and Minchin, S. D. (2004). Identification of the CRP regulon using in vitro and in vivo transcriptional profiling. Nucleic Acids Res. 32, 5874-5893.

Zhou, D., Han, Y., Qin, L., Chen, Z., Qiu, J., Song, Y., et al. (2005). Transcriptome analysis of the Mg2+-responsive PhoP regulator in Yersinia pestis. FEMS Microbiol. Lett. 250, 85-95.

Zhou, D., Han, Y., Qiu, J., Qin, L. Guo, Z., Wang, X., et al. (2006a). Genome-wide transcriptional response of Yersinia pestis to stressful conditions simulating phagolysosomal environments. Microbes Infect. 8, 2669-2678.

Zhou, D., Han, Y., and Yang, R. (2006b). Molecular and physiological insights into plague transmission, virulence and etiology. Microbes Infect. 8, 273-284.

Zhou, D., Qin, L., Han, Y., Qiu, J., Chen, Z., Li, B., et al. (2006c). Global analysis of iron assimilation and fur regulation in Yersinia pestis. FEMS Microbiol. Lett. 258, 9-17.

Zhou, D., Han, Y., Song, Y., Huang, P., and Yang, R. (2004a). Comparative and evolutionary genomics of Yersinia pestis. Microbes Infect. 6 , 1226-1234.

Zhou, D., Han, Y., Song, Y., Tong, Z., Wang, J., Guo, Z., et al. (2004b). DNA microarray analysis of genome dynamics in Yersinia pestis: insights into bacterial genome microevolution and niche adaptation. J. Bacteriol. 186, 5138-5146.

Zhou, D., Tong, Z., Song, Y., Han, Y., Pei, D., Pang, X., et al. (2004c). Genetics of metabolic variations between Yersinia pestis biovars and the proposal of a new biovar, microtus. J. Bacteriol. 186, 5147-5152.

Zhou, D., and Yang, R. (2006). Global analysis of gene transcription regulation in prokaryotes. Cell. Mol. Life Sci. 63, 2260-2290.

Zhou, D., and Yang, R. (2009). Molecular Darwinian evolution of virulence in Yersinia pestis. Infect. Immun. 77, 2242-2250.

Zhou, L., Ying, W., Han, Y., Chen, M., Yan, Y., Li, L., et al. (2012). A proteome reference map and virulence factors analysis of Yersinia pestis 91001. J. Proteomics 75, 894-907.

Zubarev, R., and Mann, M. (2007). On the proper use of mass accuracy in proteomics. Mol. Cell Proteomics 6, 377-381.

Conflict of Interest Statement: The authors declare that the research was conducted in the absence of any commercial or financial relationships that could be construed as a potential conflict of interest.

Received: 29 August 2012; accepted: 27 November 2012; published online: 13 December 2012.

Citation: Yang R, Du Z, Han Y, Zhou L, Song Y, Zhou D and Cui Y (2012) Omics strategies for revealing Yersinia pestis virulence. Front. Cell. Inf. Microbio. 2:157. doi: 10.3389/fcimb.2012.00157

Copyright (c) 2012 Yang, Du, Han, Zhou, Song, Zhou and Cui. This is an open-access article distributed under the terms of the Creative Commons Attribution License, which permits use, distribution and reproduction in other forums, provided the original authors and source are credited and subject to any copyright notices concerning any thirdparty graphics etc. 